\title{
An Extinction Study of the Taurus Dark Cloud Complex
}

\section{Citation}

Arce, Hector G., and Alyssa A. Goodman. 1999. "An Extinction Study of the Taurus Dark Cloud Complex." The Astrophysical Journal 517 (1): 264-81. https://doi.org/10.1086/307168.

\section{Permanent link}

http://nrs.harvard.edu/urn-3:HUL.InstRepos:41397446

\section{Terms of Use}

This article was downloaded from Harvard University's DASH repository, and is made available under the terms and conditions applicable to Other Posted Material, as set forth at http:// nrs.harvard.edu/urn-3:HUL.InstRepos:dash.current.terms-of-use\#LAA

\section{Share Your Story}

The Harvard community has made this article openly available.

Please share how this access benefits you. Submit a story.

\section{Accessibility}




\title{
AN EXTINCTION STUDY OF THE TAURUS DARK CLOUD COMPLEX
}

\author{
HÉCtor G. ArCE ${ }^{1}$ and Alyssa A. Goodman ${ }^{2}$ \\ Harvard-Smithsonian Center for Astrophysics,60 Garden Street, Cambridge, MA 02138; harce@cfa.harvard.edu, agoodman@cfa.harvard.edu \\ Received 1998 May 26; accepted 1998 December 29
}

\begin{abstract}
We present a study of the detailed distribution of extinction in a region of the Taurus dark cloud complex. Our study uses new $B V R$ images of the region, spectral classification data for 95 stars, and IRAS Sky Survey Atlas (ISSA) 60 and $100 \mu \mathrm{m}$ images. We study the extinction of the region in four different ways, and we present the first intercomparison of all these methods, which are as follows: (1) using the color excess of background stars for which spectral types are known, (2) using the ISSA 60 and $100 \mu \mathrm{m}$ images, (3) using star counts, and (4) using an optical ( $V$ and $R$ ) version of the average color excess method used by Lada et al. We find that all four methods give generally similar results - with important exceptions. As expected, all the methods show an increase in extinction due to dense dusty regions (i.e., dark clouds and IRAS cores) and a general increase in extinction with increasing declination, due to a larger content of dust in the northern regions of the Taurus dark cloud complex. Some of the discrepancies between the methods are caused by assuming a constant dust temperature for each line of sight in the ISSA extinction maps and not correcting for unexpected changes in the background stellar population (i.e., the presence of a cluster or Galactic gradients in the stellar density and average $V-R$ color). To study the structure in the dust distribution, we compare the ISSA extinction and the extinction measured for individual stars. From the comparison, we conclude that in the relatively lowextinction regions studied, with $0.9<A_{V}<3.0 \mathrm{mag}$ (away from filamentary dark clouds and IRAS cores), there are no fluctuations in the dust column density greater than $45 \%$ (at the $99.7 \%$ confidence level), on scales smaller than $0.2 \mathrm{pc}$. We also report the discovery of a previously unknown open cluster of stars behind the Taurus dark cloud near R.A. $4^{\mathrm{h}} 19^{\mathrm{m}}$, decl. $27^{\circ} 30^{\prime}$ (B1950).
\end{abstract}

Subject headings: dust, extinction - infrared: ISM: continuum -

ISM: individual (Taurus Dark Cloud) - techniques: photometric -

techniques: spectroscopic

\section{INTRODUCTION}

In order to understand how molecular clouds evolve and eventually produce stars, it is necessary to study the distribution of their star-forming matter. Since the clouds' main constituent, molecular hydrogen, is generally unobservable, it is necessary to use other tracers, whose abundance relative to hydrogen can be reliably estimated, to map out the distribution of material. The extinction of background starlight is the result of the absorption and scattering of photons off dust grains, so, for a given line of sight, the amount of extinction is directly proportional to the amount of dust. If the gas-to-dust ratio is known and constant (e.g., Bohlin, Savage, \& Drake 1978), then a detailed study of the dust distribution in a cloud serves as a detailed study of its mass distribution.

The study of fluctuations in the dust distribution is also interesting independent of its usefulness as a mass tracer. Strong fluctuations in the dust distribution have considerable impact on both the physics and chemistry of the interstellar medium (ISM), which both depend heavily on the extinction (opacity) structure on all scales (see Thoraval, Boissé, \& Duvert 1997, and references therein). In addition, knowledge of the spatial structure and amount of extinction in the Galactic ISM is important since it affects the apparent colors of background sources, such as stars and galaxies.

The most direct measure of reddening is the color excess of a star with known spectral type. Unfortunately, mapping

\footnotetext{
${ }^{1}$ National Science Foundation Minority Graduate Fellow.

${ }^{2}$ National Science Foundation Young Investigator.
}

out extended distributions of extinction (reddening) by obtaining photometry and a spectrum for large numbers of stars is very tedious and time consuming and usually impractical. Therefore, the traditional way of undertaking an extinction study of a fairly large region of the sky has been, until recently, through the use of optical star counts using photographic plates (Bok \& Cordwell 1973). This method can only be used up to an extinction of approximately $4 \mathrm{mag}$, with a resolution of $\sim 2.5$. Thankfully, recent advances in technology have led to the development of new methods of deriving the extinction in dark cloud regions. For example, in a study of the structure of nearby dark clouds, Wood, Myers, \& Daugherty (1994) use 60 and 100 $\mu \mathrm{m}$ images taken by $I R A S$ to calculate $100 \mu \mathrm{m}$ optical depth, from which they obtain the extinction $\left(A_{V}\right)$. Lada et al. (1994, hereafter LLCB) took advantage of the improvements in infrared array cameras to devise a clever new method of measuring extinction. The LLCB technique, ${ }^{3}$ which combines measurements of near-infrared ( $H$ and $K$ ) color excess and certain techniques of star counting, has a higher angular resolution and can probe greater optical depths than those achieved by optical star counting alone.

In this study we use four different methods of measuring $A_{V}$, using (1) the color excess of individual background stars for which we could obtain spectral types, (2) ISSA 60 and $100 \mu \mathrm{m}$ images to estimate dust opacity, (3) traditional star counting, and (4) an optical ( $V$ and $R$ ) version of the average color excess method used by Lada et al. (1994). To our

\footnotetext{
${ }^{3}$ This technique has been called the "NICE" (near-infrared color excess) method by Alves et al. (1998).
} 
knowledge, this is the first time that all of these different methods have been directly intercompared. We describe the acquisition and reduction of the data in $\S 2$. In $\S 3$ we present the results of the observations, and in $\S 4$ we offer analysis and discussion. Readers interested primarily in intercomparison of the various methods and limits on density fluctuations should skim $\S \S 2$ and 3 and focus more on $\S \S 4$ and 5 . In $\S 5$ we compare and rate the four different methods of obtaining $A_{V}$. We devote $\S 6$ to our conclusions.

\section{DATA}

The new photometric and spectroscopic observations used in this paper were originally obtained to conduct the polarization-extinction study described in Arce et al. (1998). The photometry consists of $B, V$, and $R C C D$ images of two $10^{\prime}$ by $\sim 5^{\circ}$ "cuts" through the Taurus dark cloud complex (see Fig. 1). In the spectroscopic observations, we observed 95 stars (most of them in cut 1), in order to determine their

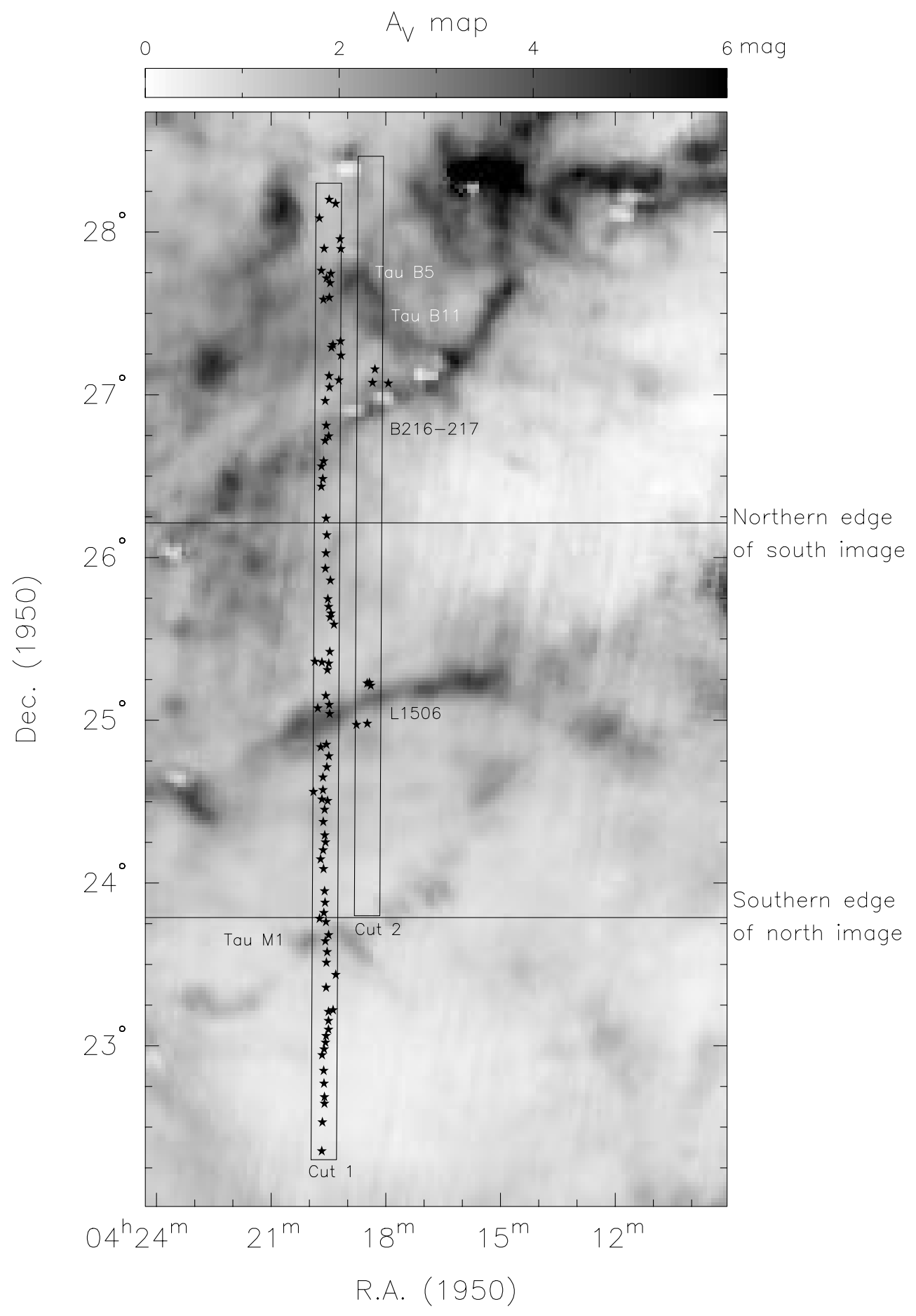

FIG. 1.-Extinction $\left(A_{V}\right)$ map of part of the Taurus dark cloud region. The map was obtained using the method described in $\S 2.3$. The two cuts for which we have photometric (CCD) data are shown. The two filamentary dark clouds L1506 and B216-217 and the three IRAS cores Tau M1, Tau B5, and Tau B11 are identified. The star symbols represent the position of the stars that were classified by their spectra. The horizontal lines mark the northern and southern edges of the two images that were used to make this map. 
spectral types. The cuts shown in Figure 1 pass through two well-known filamentary dark clouds (L1506 and B216-217, both at a distance of $140 \mathrm{pc}$ from the Sun), as well as very low extinction regions, giving our photometric observations a fairly large dynamic range in extinction. In the spectroscopic observations, we selected our target stars along the two cuts by virtue of their relative brightness, in that we attempted to exclude foreground stars by not selecting stars that appear unusually bright. Table 1 lists the spectral type, apparent $V$ magnitude and $B-V$ color, and the derived spectroscopic parallax distance for each target star. Our stellar sample has only one star with a distance less than $140 \mathrm{pc}$, which confirms that we largely succeeded in excluding foreground stars. In addition to the new photometric and spectroscopic observations we also obtained coadded images of flux density from the IRAS Sky Survey Atlas (ISSA), in order to examine the far-infrared emission from dust in the region.

\subsection{Photometric Data}

The broadband imaging data of the two cuts (Fig. 1) through the Taurus dark cloud complex were obtained using the Smithsonian Astrophysical Observatory (SAO) AndyCam on the Fred Lawrence Whipple Observatory (FLWO) $1.2 \mathrm{~m}$ telescope on Mount Hopkins, Arizona. AndyCam is a camera with a thinned backside illuminated AR coated Loral $2048 \times 2048$ CCD chip. All the frames were taken in $2 \times 2$ bin mode, giving a plate scale of 0 ".63 pixel $^{-1}$. In 1995 November, a total of 64 frames in different positions in the sky were taken in the $B, V$, and $R$ bands, where $R$ is the Cousins $R$-band filter with an effective wavelength equal to $0.64 \mu \mathrm{m}$. In each position we obtained one $200 \mathrm{~s}$ exposure for each broadband filter. Each telescope pointing was a little less than $10^{\prime}$ north of the previous position, and since each frame is slightly larger than $10^{\prime} \times 10^{\prime}$, there is a small sky overlap between the frames successive positions. The first cut extends from declination of $22^{\circ} 30^{\prime}$ to $28^{\circ} 20^{\prime}$, centered on right ascension $4^{\mathrm{h}} 22^{\mathrm{m}} 36^{\mathrm{s}}$ (J2000), with a total of 36 frames. The second cut extends from declination $24^{\circ} 00^{\prime}$ to $28^{\circ} 30^{\prime}$, centered on right ascension $4^{\mathrm{h}} 21^{\mathrm{m}} 29^{\mathrm{s}}(\mathrm{J} 2000)$, with a total of 28 frames. In addition to the frames acquired in 1995 November, 17 frames were taken in the $U, B$, and $V$ bands in 1996 October, four frames were taken in the $B$ and $V$ bands in 1996 November, and two frames were taken in the $B$ and $V$ bands in 1997 March - all with the same instrument configuration as the 1995 November frames. The additional frames were taken because not all of the original frames were of good quality; several frames were of regions of special interest around the two dark clouds peripheries (outside the cuts), which the original frames did not include, and because shorter exposure images of some of the regions covered by the original frames were needed. The exposure times were 80,150 , and $200 \mathrm{~s}$ for $V, B$, and $U$, respectively, for frames of new sky positions, and $30 \mathrm{~s}$ in $V, 50 \mathrm{~s}$ in $B$, and $100 \mathrm{~s}$ in $U$ for frames with repeated positions in the sky.

All of the stellar photometric data reduction was accomplished using standard Image Reduction and Analysis Facility (IRAF) routines. For stars whose spectra had been measured (see $\S 2.2$ ), photometry was obtained in the flatfielded, background-subtracted images using the APPHOT routine. After analyzing the dependence of magnitude value with aperture size in the standard stars for all nights, it was decided to use an aperture radius of 14 pixels. With this aperture size, less than $10 \%$ of the stars in the most crowded field, with $R$-band apparent magnitude $\left(m_{R}\right)$ between 14.5 and $18.0 \mathrm{mag}$, have neighbors within the aperture. The correction to the standard photometric system was accomplished using Landolt standards (Landolt 1992). A set of standards were observed for each night, at different times of the night, at varying air masses. These were then used to solve a set of linear equations that would give the stars' $V$ magnitude, $B-V$ and $V-R$ colors using the routines in the IRAF package PHOTCAL. The errors obtained from the APPHOT routine and the errors in the transformation equation fit were summed in quadrature to give the final errors in the photometry. These were \pm 0.02 to $0.06 \mathrm{mag}$ for $V$ and $B-V$ for stars with $V$ between 12.3 and $17.4 \mathrm{mag}$. We calculated the photometry of the standard stars used to derive the transformation equation and compared our results with those quoted by Landolt (1992). By doing so we convinced ourselves that the values of our final $1 \sigma$ errors (Table 1) are a reasonably good estimation of the true photometry uncertainties.

In addition to obtaining apparent magnitudes and colors for stars, the photometric data were also used to do a star count of the region. The routine DAOFIND was used to detect sources in the $R$ filter frames. This routine automatically detects objects that are above a certain intensity threshold, and within a limit of sharpness and roundness, all of which the user specifies. We used a finding threshold of 5 times the rms sky noise in each frame. The objects detected by the routine are then stored in a file with the objects' coordinates. Although care was taken to select a limit of sharpness and roundness so that DAOFIND would only detect stars, other objects (like cosmic rays and galaxies) were also detected, and some stars clearly above the threshold level were not detected. Thus, the $R$ frames were painstakingly inspected visually to erase detected objects that were not stars and add the few stars that were clearly above the threshold but were not originally detected. We obtained the photometry of all the stars in the sample and then made a histogram (Fig. 2) of the number of stars versus apparent $R$ magnitude in order to study the completeness of the sample. From Figure 2 we estimate the upper completeness

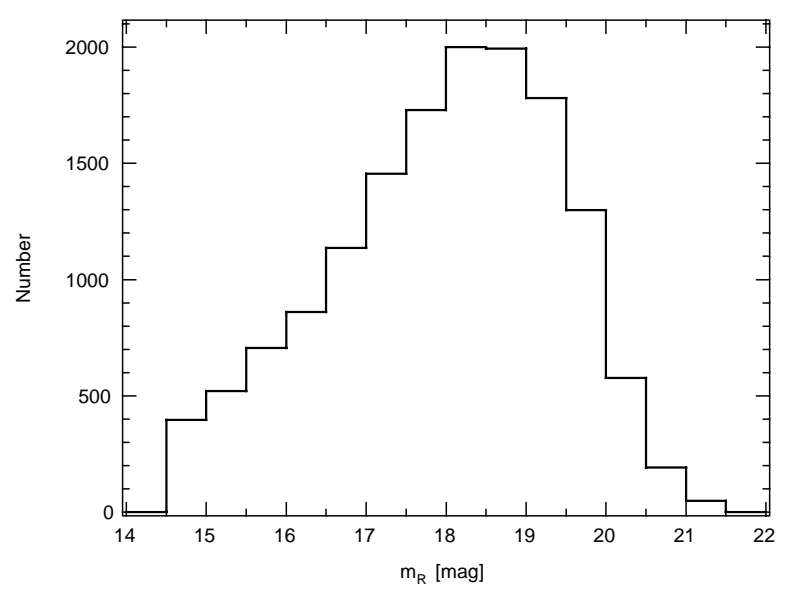

FIG. 2.-Histogram of the distribution of stars with respect to apparent $R$-band magnitude $\left(m_{R}\right)$ is shown. These are the stars detected in the 64 $R$-band frames taken in 1995 November. Stars with $m_{R}<14.5$ are saturated in the $200 \mathrm{~s}$ exposures, and thus we do not include them in the distribution. From this figure we estimate our sample is at least $90 \%$ complete for $14.5 \leq m_{R}<18.0$. 
TABLE 1

Photometric and SpeCtroscopic Data

\begin{tabular}{|c|c|c|c|c|c|c|c|c|c|c|c|c|}
\hline Star Name & $\begin{array}{l}\text { R.A. } \\
\text { (J2000) }\end{array}$ & $\begin{array}{l}\text { Decl. } \\
\text { (J2000) }\end{array}$ & $\begin{array}{c}V \\
(\mathrm{mag})\end{array}$ & $\begin{array}{c}\sigma(V) \\
(\mathrm{mag})\end{array}$ & $\begin{array}{l}B-V \\
(\mathrm{mag})\end{array}$ & $\begin{array}{c}\sigma(B-V) \\
(\mathrm{mag})\end{array}$ & Spectral Type & $\begin{array}{l}E_{B-V} \\
\text { (mag) }\end{array}$ & $\begin{array}{c}A_{V} \\
\text { (mag) }\end{array}$ & $\begin{array}{l}\sigma\left(A_{V}\right) \\
\text { (mag) }\end{array}$ & $\begin{array}{l}\text { Distance } \\
\quad(\mathrm{pc})\end{array}$ & $\begin{array}{l}\sigma(d) \\
(\mathrm{pc})\end{array}$ \\
\hline $011005 \ldots \ldots$ & 42239.3 & 222808 & 14.94 & 0.02 & 0.58 & 0.03 & $\mathrm{~F} 2$ & 0.23 & 0.73 & 0.16 & 1669 & 261 \\
\hline $011003 \ldots \ldots$ & 42238.5 & 223847 & 16.57 & 0.02 & 0.97 & 0.04 & G8 & 0.24 & 0.75 & 0.22 & 1156 & 291 \\
\hline $011001 \ldots \ldots$ & 42235.5 & 224539 & 14.87 & 0.02 & 0.62 & 0.03 & F6 & 0.16 & 0.51 & 0.16 & 1295 & 202 \\
\hline $021015 \ldots \ldots$ & 42235.5 & 224802 & 15.99 & 0.02 & 0.83 & 0.03 & F8 & 0.31 & 0.97 & 0.18 & 1459 & 235 \\
\hline $021014 \ldots \ldots$ & 42236.2 & 225301 & 15.81 & 0.02 & 1.07 & 0.03 & G8 & 0.34 & 1.05 & 0.21 & 711 & 177 \\
\hline $021013 \ldots \ldots$ & 42236.9 & 225746 & 15.41 & 0.02 & 0.69 & 0.06 & F6 & 0.23 & 0.72 & 0.23 & 1511 & 262 \\
\hline $021012 \ldots \ldots$. & 42239.5 & 230330 & 14.94 & 0.02 & 0.71 & 0.03 & F7 & 0.21 & 0.64 & 0.16 & 955 & 149 \\
\hline $021011 \ldots \ldots$ & 42236.3 & 230539 & 15.18 & 0.02 & 0.74 & 0.03 & F7 & 0.24 & 0.73 & 0.16 & 1231 & 192 \\
\hline 00N5.2 ....... & 42234.4 & 230809 & 14.40 & 0.02 & 0.58 & 0.02 & F3 & 0.21 & 0.65 & 0.15 & 1292 & 199 \\
\hline $031025 \ldots \ldots$ & 42233.4 & 231038 & 15.37 & 0.02 & 0.84 & 0.03 & G0 & 0.26 & 0.81 & 0.18 & 1075 & 263 \\
\hline $031024 \ldots \ldots$ & 42229.7 & 231254 & 15.47 & 0.02 & 0.90 & 0.03 & G4 & 0.26 & 0.79 & 0.19 & 861 & 212 \\
\hline $031023 \ldots \ldots$ & 42229.7 & 231611 & 15.23 & 0.02 & 0.69 & 0.03 & F6 & 0.23 & 0.70 & 0.16 & 1398 & 219 \\
\hline $031022 \ldots \ldots$. & 42229.9 & 231932 & 16.34 & 0.02 & 0.94 & 0.03 & F9 & 0.39 & 1.21 & 0.18 & 1462 & 236 \\
\hline TDC071..... & 42222.5 & 232007 & 15.60 & 0.02 & 0.54 & 0.03 & A9 & 0.27 & 0.82 & 0.17 & 2720 & 434 \\
\hline $041035 \ldots \ldots$ & 42233.7 & 232824 & 14.57 & 0.04 & 0.78 & 0.07 & F7 & 0.28 & 0.86 & 0.25 & 877 & 158 \\
\hline TDC085...... & 42218.6 & 233310 & 16.25 & 0.05 & 0.81 & 0.07 & F6 & 0.34 & 1.07 & 0.25 & 1884 & 342 \\
\hline $041033 \ldots \ldots$ & 42233.6 & 233738 & 16.23 & 0.04 & 1.17 & 0.06 & G5 & 0.51 & 1.59 & 0.27 & 809 & 212 \\
\hline $041032 \ldots \ldots$ & 42232.4 & 234130 & 15.01 & 0.04 & 0.98 & 0.06 & F7 & 0.48 & 1.50 & 0.23 & 798 & 139 \\
\hline $041031 \ldots \ldots$ & 42235.3 & 234531 & 16.16 & 0.04 & 1.50 & 0.06 & F8 & 0.99 & 3.05 & 0.25 & 605 & 109 \\
\hline $051045 \ldots \ldots$ & 42230.2 & 234747 & 15.15 & 0.04 & 1.07 & 0.06 & F5 & 0.65 & 2.02 & 0.23 & 805 & 140 \\
\hline $051044 \ldots \ldots$ & 42234.6 & 235236 & 14.90 & 0.04 & 0.81 & 0.06 & F3 & 0.44 & 1.38 & 0.23 & 1163 & 202 \\
\hline TDC101...... & 42244.6 & 235349 & 15.51 & 0.04 & 0.75 & 0.06 & F5 & 0.33 & 1.02 & 0.23 & 1508 & 263 \\
\hline $051043 \ldots \ldots$ & 42237.8 & 235557 & 16.08 & 0.02 & 0.77 & 0.03 & F6 & 0.31 & 0.96 & 0.16 & 1839 & 288 \\
\hline $051042 \ldots \ldots$. & 42236.0 & 235945 & 15.02 & 0.02 & 0.79 & 0.02 & F6 & 0.33 & 1.01 & 0.15 & 1103 & 170 \\
\hline $051041 \ldots \ldots$ & 42236.8 & 240360 & 14.37 & 0.02 & 0.90 & 0.02 & F9 & 0.35 & 1.08 & 0.17 & 626 & 99 \\
\hline $061055 \ldots \ldots$ & 42238.7 & 241210 & 16.43 & 0.04 & 0.84 & 0.06 & F6 & 0.38 & 1.19 & 0.22 & 1943 & 334 \\
\hline $061054 \ldots \ldots$ & 42243.4 & 241544 & 15.29 & 0.04 & 0.87 & 0.05 & F7 & 0.37 & 1.14 & 0.21 & 1072 & 181 \\
\hline $061053 \ldots \ldots$ & 42239.6 & 241904 & 14.94 & 0.04 & 0.74 & 0.05 & F0 & 0.43 & 1.32 & 0.21 & 1530 & 259 \\
\hline $061052 \ldots \ldots$ & 42235.9 & 242158 & 15.83 & 0.04 & 1.03 & 0.06 & G1 & 0.43 & 1.34 & 0.24 & 951 & 243 \\
\hline $061051 \ldots \ldots$ & 42237.1 & 242434 & 15.09 & 0.04 & 1.42 & 0.05 & G9 & 0.64 & 1.98 & 0.26 & 302 & 78 \\
\hline $071065 \ldots \ldots$ & 42239.4 & 242930 & 16.04 & 0.04 & 1.02 & 0.06 & F7 & 0.52 & 1.63 & 0.22 & 1207 & 208 \\
\hline $071064 \ldots \ldots$ & 42237.6 & 243402 & 12.93 & 0.02 & 0.86 & 0.02 & F5 & 0.44 & 1.37 & 0.15 & 390 & 60 \\
\hline $071063 \ldots \ldots$ & 42232.9 & 243716 & 14.63 & 0.04 & 0.95 & 0.05 & F9 & 0.40 & 1.25 & 0.23 & 655 & 114 \\
\hline $\mathrm{N} 14.51 \ldots \ldots$. & 42241.9 & 243749 & 14.15 & 0.02 & 0.77 & 0.02 & A9 & 0.50 & 1.57 & 0.16 & 991 & 155 \\
\hline $\mathrm{N} 14.52 \ldots \ldots$. & 42258.2 & 244035 & 15.25 & 0.02 & 0.78 & 0.03 & F6 & 0.32 & 1.00 & 0.16 & 1231 & 192 \\
\hline $071062 \ldots \ldots$. & 42240.3 & 244114 & 12.82 & 0.02 & 1.34 & 0.02 & G9 & 0.56 & 1.74 & 0.30 & 199 & 32 \\
\hline $071061 \ldots \ldots$ & 42240.3 & 244556 & 15.14 & 0.04 & 0.85 & 0.05 & F3 & 0.48 & 1.49 & 0.21 & 1231 & 208 \\
\hline $081075 \ldots \ldots$ & 42234.3 & 244939 & 15.40 & 0.04 & 1.31 & 0.05 & G9 & 0.53 & 1.65 & 0.26 & 406 & 105 \\
\hline $081074 \ldots \ldots$. & 42231.2 & 245344 & 15.78 & 0.04 & 0.93 & 0.05 & F0 & 0.62 & 1.93 & 0.21 & 1703 & 288 \\
\hline AG-136 ...... & 42244.0 & 245704 & 12.32 & 0.02 & 0.93 & 0.02 & F7 & 0.43 & 1.33 & 0.15 & 249 & 38 \\
\hline $081073 \ldots \ldots$ & 42235.2 & 245759 & 15.66 & 0.04 & 1.12 & 0.05 & F7 & 0.62 & 1.93 & 0.21 & 883 & 149 \\
\hline AG-133 ...... & 42148.0 & 250520 & 14.15 & 0.03 & 0.91 & 0.04 & F7 & 0.41 & 1.27 & 0.18 & 596 & 96 \\
\hline AG-132 ...... & 42131.0 & 250548 & 14.17 & 0.02 & 1.14 & 0.02 & G1 & 0.54 & 1.67 & 0.19 & 379 & 93 \\
\hline $091085 \ldots \ldots$ & 42230.3 & 250919 & 16.96 & 0.04 & 2.12 & 0.09 & G0 & 1.54 & 4.78 & 0.33 & 358 & 99 \\
\hline AG-102 ...... & 42249.0 & 251123 & 12.29 & 0.03 & 1.27 & 0.10 & A9 & 1.00 & 3.10 & 0.34 & 207 & 43 \\
\hline $091084 \ldots \ldots$. & 42231.0 & 251240 & 15.91 & 0.04 & 1.36 & 0.06 & G3 & 0.73 & 2.27 & 0.24 & 586 & 150 \\
\hline $091083 \ldots \ldots$. & 42236.5 & 251557 & 16.32 & 0.04 & 1.23 & 0.06 & G9 & 0.45 & 1.41 & 0.27 & 696 & 182 \\
\hline $092087 \ldots \ldots$. & 42126.0 & 251949 & 15.75 & 0.02 & 0.85 & 0.03 & $\mathrm{~A} 2$ & 0.79 & 2.45 & 0.17 & 2158 & 343 \\
\hline 00Ld.1 ........ & 42131.5 & 252039 & 15.41 & 0.02 & 0.53 & 0.03 & $\mathrm{~A} 2$ & 0.47 & 1.46 & 0.17 & 2949 & 469 \\
\hline AG-105 ...... & 42128.0 & 252051 & 15.16 & 0.03 & 1.29 & 0.04 & G9 & 0.51 & 1.58 & 0.24 & 376 & 96 \\
\hline $091081 \ldots \ldots$. & 42234.2 & 252532 & 14.22 & 0.02 & 0.78 & 0.02 & F5 & 0.36 & 1.11 & 0.15 & 795 & 123 \\
\hline $101095 \ldots \ldots$ & 42232.0 & 252751 & 15.27 & 0.02 & 0.85 & 0.03 & F5 & 0.43 & 1.34 & 0.16 & 1162 & 182 \\
\hline 0N20.1 ....... & 42243.0 & 252818 & 16.14 & 0.03 & 0.78 & 0.05 & F6 & 0.32 & 1.00 & 0.19 & 1854 & 304 \\
\hline 0N20.2 ....... & 42258.9 & 252832 & 16.34 & 0.03 & 0.40 & 0.05 & $\mathrm{~A} 2$ & 0.34 & 1.05 & 0.20 & 5460 & 911 \\
\hline $101094 \ldots \ldots$ & 42230.5 & 253213 & 13.61 & 0.02 & 0.89 & 0.02 & F6 & 0.43 & 1.35 & 0.15 & 493 & 76 \\
\hline $101092 \ldots \ldots$ & 42224.3 & 254215 & 15.55 & 0.02 & 1.01 & 0.04 & F7 & 0.51 & 1.57 & 0.17 & 986 & 157 \\
\hline $101091 \ldots \ldots$ & 42230.3 & 254458 & 13.27 & 0.02 & 0.74 & 0.02 & A8 & 0.50 & 1.57 & 0.16 & 692 & 108 \\
\hline 0N22.1 $\ldots \ldots$. & 42228.9 & 254624 & 14.61 & 0.02 & 0.64 & 0.02 & A5 & 0.50 & 1.55 & 0.16 & 1553 & 244 \\
\hline $111105 \ldots \ldots$ & 42232.8 & 254849 & 16.85 & 0.02 & 0.64 & 0.04 & A6 & 0.48 & 1.49 & 0.18 & 4279 & 691 \\
\hline $111104 \ldots \ldots$ & 42234.0 & 255139 & 14.83 & 0.02 & 0.88 & 0.02 & F5 & 0.46 & 1.41 & 0.15 & 918 & 142 \\
\hline $111102 \ldots \ldots$ & 42230.2 & 255831 & 15.90 & 0.02 & 1.24 & 0.03 & G9 & 0.46 & 1.43 & 0.22 & 567 & 142 \\
\hline $111101 \ldots \ldots$ & 42238.2 & 260253 & 12.76 & 0.05 & 1.18 & 0.10 & G1 & 0.58 & 1.80 & 0.35 & 186 & 52 \\
\hline $121115 \ldots \ldots$ & 42237.4 & 260834 & 14.27 & 0.04 & 1.17 & 0.06 & G1 & 0.57 & 1.78 & 0.22 & 378 & 97 \\
\hline $121113 \ldots \ldots$ & 42236.1 & 261512 & 15.64 & 0.04 & 0.93 & 0.06 & F6 & 0.47 & 1.45 & 0.22 & 1197 & 206 \\
\hline 121112 & 42237.5 & 262120 & 16.03 & 0.04 & 1.18 & 0.06 & G8 & 0.45 & 1.41 & 0.27 & 665 & 174 \\
\hline
\end{tabular}


TABLE 1-Continued

\begin{tabular}{|c|c|c|c|c|c|c|c|c|c|c|c|c|}
\hline Star Name & $\begin{array}{l}\text { R.A. } \\
\text { (J2000) }\end{array}$ & $\begin{array}{l}\text { Decl. } \\
\text { (J2000) }\end{array}$ & $\begin{array}{c}V \\
(\mathrm{mag})\end{array}$ & $\begin{array}{c}\sigma(V) \\
(\mathrm{mag})\end{array}$ & $\begin{array}{l}B-V \\
\text { (mag) }\end{array}$ & $\begin{array}{c}\sigma(B-V) \\
(\mathrm{mag})\end{array}$ & Spectral Type & $\begin{array}{l}E_{B-V} \\
(\mathrm{mag})\end{array}$ & $\begin{array}{c}A_{V} \\
(\mathrm{mag})\end{array}$ & $\begin{array}{l}\sigma\left(A_{V}\right) \\
(\mathrm{mag})\end{array}$ & $\begin{array}{l}\text { Distance } \\
\text { (pc) }\end{array}$ & $\begin{array}{l}\sigma(d) \\
(\mathrm{pc})\end{array}$ \\
\hline $131124 \ldots \ldots$ & 42245.2 & 263304 & 15.09 & 0.04 & 1.12 & 0.06 & G0 & 0.54 & 1.66 & 0.24 & 639 & 163 \\
\hline $131123 \ldots \ldots$ & 42242.9 & 263557 & 15.37 & 0.04 & 1.23 & 0.06 & G3 & 0.60 & 1.85 & 0.26 & 554 & 144 \\
\hline $131122 \ldots \ldots$ & 42245.2 & 264032 & 16.44 & 0.04 & 1.29 & 0.07 & F7 & 0.79 & 2.44 & 0.26 & 1002 & 183 \\
\hline $131121 \ldots \ldots$ & 42241.8 & 264228 & 15.61 & 0.04 & 0.96 & 0.06 & F3 & 0.59 & 1.83 & 0.23 & 1307 & 228 \\
\hline $141135 \ldots \ldots$. & 42239.7 & 265000 & 15.79 & 0.02 & 1.24 & 0.03 & F7 & 0.74 & 2.29 & 0.16 & 795 & 124 \\
\hline $141134 \ldots \ldots$ & 42233.9 & 265138 & 14.29 & 0.02 & 1.34 & 0.02 & A5 & 1.20 & 3.73 & 0.16 & 492 & 77 \\
\hline $141133 \ldots \ldots$ & 42238.1 & 265537 & 15.08 & 0.02 & 0.94 & 0.03 & F3 & 0.57 & 1.76 & 0.15 & 1056 & 163 \\
\hline $141131 \ldots \ldots$ & 42239.8 & 270444 & 16.05 & 0.02 & 0.86 & 0.04 & F4 & 0.47 & 1.47 & 0.17 & 1722 & 273 \\
\hline $151145 \ldots \ldots$ & 42233.1 & 270943 & 17.32 & 0.03 & 1.06 & 0.05 & F7 & 0.56 & 1.73 & 0.19 & 2074 & 341 \\
\hline 00B $2.2 \ldots \ldots \ldots$ & 42125.5 & 271131 & 13.89 & 0.02 & 0.72 & 0.02 & $\mathrm{~A} 2$ & 0.66 & 2.03 & 0.16 & 1125 & 176 \\
\hline 00B1.2 ........ & 42100.7 & 271116 & 16.01 & 0.03 & 0.58 & 0.04 & $\mathrm{~F} 1$ & 0.25 & 0.79 & 0.18 & 2916 & 470 \\
\hline TDC301...... & 42218.4 & 271220 & 17.35 & 0.03 & 1.16 & 0.05 & G4 & 0.52 & 1.60 & 0.25 & 1412 & 364 \\
\hline TDC304 ....... & 42234.0 & 271355 & 15.50 & 0.02 & 1.13 & 0.03 & F9 & 0.58 & 1.79 & 0.19 & 761 & 124 \\
\hline 00B2.1 ........ & 42122.0 & 271628 & 13.01 & 0.02 & 0.47 & 0.02 & $\mathrm{~A} 1$ & 0.44 & 1.35 & 0.16 & 1179 & 185 \\
\hline TDC312...... & 42211.5 & 272125 & 15.72 & 0.02 & 0.92 & 0.03 & F6 & 0.46 & 1.44 & 0.16 & 1247 & 195 \\
\hline $151141 \ldots \ldots$ & 42230.4 & 272423 & 16.62 & 0.02 & 1.40 & 0.04 & G4 & 0.76 & 2.37 & 0.22 & 708 & 178 \\
\hline TDC321...... & 42228.2 & 272522 & 15.29 & 0.02 & 1.20 & 0.03 & A9 & 0.93 & 2.87 & 0.17 & 919 & 146 \\
\hline TDC323...... & 42215.2 & 272641 & 16.67 & 0.02 & 1.03 & 0.04 & G3 & 0.40 & 1.23 & 0.20 & 1342 & 333 \\
\hline $161152 \ldots \ldots$ & 42243.4 & 274202 & 14.42 & 0.02 & 1.03 & 0.03 & A8 & 0.80 & 2.47 & 0.17 & 776 & 123 \\
\hline TDC332...... & 42234.2 & 274245 & 16.15 & 0.02 & 1.17 & 0.03 & F5 & 0.75 & 2.32 & 0.16 & 1110 & 174 \\
\hline $171165 \ldots \ldots$ & 42233.0 & 274809 & 14.61 & 0.02 & 1.36 & 0.03 & G5 & 0.70 & 2.17 & 0.21 & 294 & 73 \\
\hline TDC342...... & 42238.7 & 274955 & 15.35 & 0.02 & 0.92 & 0.03 & $\mathrm{~F} 2$ & 0.57 & 1.76 & 0.16 & 1250 & 196 \\
\hline $171164 \ldots \ldots$ & 42231.9 & 275136 & 15.52 & 0.02 & 1.41 & 0.03 & G5 & 0.75 & 2.32 & 0.21 & 417 & 104 \\
\hline TDC341..... & 42247.1 & 275242 & 15.78 & 0.02 & 0.83 & 0.03 & F0 & 0.52 & 1.60 & 0.16 & 1974 & 310 \\
\hline TDC351..... & 42215.5 & 280046 & 15.48 & 0.02 & 0.82 & 0.03 & F4 & 0.43 & 1.33 & 0.16 & 1415 & 221 \\
\hline TDC354 ...... & 42242.8 & 280048 & 15.89 & 0.02 & 0.99 & 0.03 & F6 & 0.53 & 1.64 & 0.16 & 1230 & 192 \\
\hline TDC361 ...... & 42216.9 & 280422 & 15.12 & 0.02 & 0.80 & 0.03 & F1 & 0.47 & 1.44 & 0.16 & 1431 & 224 \\
\hline TDC364 ...... & 42250.4 & 281200 & 16.25 & 0.02 & 1.15 & 0.03 & G6 & 0.47 & 1.46 & 0.22 & 825 & 208 \\
\hline TDC373...... & 42225.0 & 281725 & 15.07 & 0.02 & 0.81 & 0.03 & F4 & 0.42 & 1.29 & 0.16 & 1192 & 186 \\
\hline $181172 \ldots \ldots$ & 42235.2 & 281855 & 15.37 & 0.02 & 0.80 & 0.03 & F7 & 0.30 & 0.94 & 0.16 & 1222 & 191 \\
\hline
\end{tabular}

NoтE.- Units of right ascension are hours, minutes, and seconds, and units of declination are degrees, arcminutes, and arcseconds.

limit to be $m_{R} \approx 18.0 \mathrm{mag}$. Stars brighter than $14.5 \mathrm{mag}$ in the $R$-band $200 \mathrm{~s}$ exposures are saturated; thus, the photometry of stars with $m_{R}<14.5$ is unreliable. Therefore we estimate that our sample is at least $90 \%$ complete for stars with $14.5 \leq m_{R}<18.0$.

\subsection{Spectroscopic Data}

The spectra of 95 stars along the cuts were obtained using the SAO FAST spectrograph on the FLWO $1.5 \mathrm{~m}$ telescope on Mount Hopkins, Arizona. The observations were carried out during the fall trimesters of 1995 and 1996. FAST was used with a $3^{\prime \prime}$ slit and a 300 line $\mathrm{mm}^{-1}$ grating. This resulted in a resolution of $\sim 6 \AA$, a spectral coverage of $\sim 3800 \AA$ (from approximately 3600 to around $7400 \AA$ ), a dispersion of $1.47 \AA$ pixel $^{-1}$, and 1.64 pixels $\operatorname{arcsec}^{-1}$ along the dispersion axis.

The spectrum of each star was used to derive its spectral type. In order to spectroscopically classify the stars, we followed O'Connell (1973) and Kenyon, Dobrzycka, \& Hartman (1994) and computed several absorption-line indices from the spectra:

$$
I_{\lambda}=-2.5 \log \left[\frac{F\left(\lambda_{2}\right)}{F^{\prime}\left(\lambda_{2}\right)}\right]
$$

where

$$
F^{\prime}\left(\lambda_{2}\right)=F\left(\lambda_{1}\right)+\left[F\left(\lambda_{3}\right)-F\left(\lambda_{1}\right)\right]\left[\frac{\lambda_{2}-\lambda_{1}}{\lambda_{3}-\lambda_{1}}\right]
$$

is the interpolated continuum flux at the feature, $\lambda_{1}$ and $\lambda_{3}$ are continuum wavelengths, $\lambda_{2}$ is the feature wavelength, and $F\left(\lambda_{i}\right)$ is the average flux in ergs $\mathrm{cm}^{-2} \mathrm{~s}^{-1} \AA^{-1}$ over a bandwidth specified in Table 2 of O'Connell (1973). We

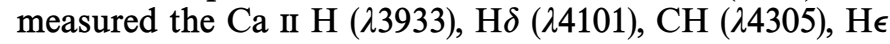
( 24340$), \mathrm{H} \zeta(\lambda 4861)$, and $\mathrm{Mg} \mathrm{I}(25175)$ indices of our program stars and then compared them to the indices of main-sequence stars in the Jacoby, Hunter, \& Christian (1984) atlas. The indices have errors of $5 \%-10 \%$ depending on the signal to noise of the spectrum. This method resulted in spectral classification of most of the stars observed with accuracy of $\sim \pm 1-2$ subclasses for spectral types A through $F$ and $\pm 2-4$ subclasses for stars with spectral type G. Stars with spectral types earlier than $\mathrm{A} 0$ were not found, and stars later than G9 were not included in the sample due to the low accuracy in their spectral classification and reddening corrections. All the stars were assumed to be main-sequence stars (luminosity type V). Kenyon et al. (1994) estimate, and also obtain, that $\sim 10 \%$ of their magnitude-limited sample of A-F stars in the Taurus region are giants. If we use this result with our magnitude-limited sample it would mean that only $\sim 7$ stars of our $69 \mathrm{~A}-\mathrm{F}$ stars are giants. In addition, the intrinsic $B-V$ color of main-sequence $\mathrm{A}$ and $\mathrm{F}$ stars differs by less than 0.05 mag from the intrinsic $B-V$ of luminosity type III and type II stars. Of the G stars, no more than five out of a total of 26 should be giant stars (Mihalas \& Binney 1981, Tables 4-9). Hence we are not introducing large errors in the extinction of each star by assuming that all of the stars we classified are luminosity type V. 
Once each star was classified, its reddening was calculated. Intrinsic $B-V$ values for each spectral type were obtained from Table A5 in Kenyon \& Hartmann (1995). The observed $B-V$ value, from the photometric study, was then used to obtain the color excess: $E_{B-V}=(B-V)-(B$ $-V)_{0}$, where $(B-V)$ is the observed color index and $(B$ $-V)_{0}$ is the unreddened intrinsic color of the star. An error in the stellar classification of $\pm 1-2$ subclasses in A-F stars transforms into an error of $\pm 0.04-0.05 \mathrm{mag}$ in $E_{B-V}$, whereas an error in the stellar classification of $\pm 2-4$ subclasses in G stars transforms into an error of $\pm 0.05-0.08$ mag in $E_{B-V}$. We assumed that $A_{V}=R_{V} E_{B-V}$, with $R_{V}$ (the ratio of total-to-selective extinction) equal to 3.1 (Savage \& Mathis 1979; Vrba \& Rydgren 1985) - the validity of this assumption is discussed below. Using absolute magnitude values for each spectral type from Lang (1992), we then obtained distances for each star (see Table 1).

When we calculated the extinction to each star using a constant value of $R_{V}=3.1$, we made the implicit assumption that the ratio of total-to-selective extinction is constant for different lines of sight. In fact, $R_{V}$ varies along different lines of sight in the Galaxy and only has a mean of $\sim 3.1$ (Savage \& Mathis 1979). In contrast with other regions, the Taurus-Auriga molecular cloud complex seems to have a fairly constant interstellar reddening law with $R_{V} \approx 3.1$ through most of the region (Vrba \& Rydgren 1985; Kenyon et al. 1994). Our $B V R$ photometry is not ample enough to derive $R_{V}$ for each line of sight. We would need observations at shorter wavelengths to be able to independently obtain the value of the ratio of total-to-selective extinction for each line of sight we observed. Thus we decided to use the ISM (and Taurus) average of $R_{V}=3.1$ and to caution the reader that we do not take the errors caused by assuming a constant $R_{V}$ into account when we calculate the errors in $A_{V}$. In $\S 3.1$ we show how the ISSA data confirm that $R_{V}=3.1$ is a good estimate of the ratio of total-toselective extinction for our region.

\subsection{ISSA Data}

The IRAS Sky Survey Atlas (ISSA) was used to obtain images of flux density at 60 and $100 \mu \mathrm{m}$ of the Taurus dark cloud complex. Our region of interest lies in two different (but overlapping) ISSA fields. Each of these is a $500 \times 500$ pixel image, covering a 12.5 by 12.5 field of sky with a pixel size of 1'.5. The maps have units of $\mathrm{MJy} \mathrm{Sr}^{-1}$, are made with gnomic projection, have spatial resolution smoothed to the IRAS beam at $100 \mu \mathrm{m}$ (approximately $5^{\prime}$ ), and the zodiacal emission has been removed from them. The 12.5 by 12.5 images were cropped in order to keep only the region of the Taurus dark cloud complex shown in Figure 1. This resulted in a total of four different images: two (60 and 100 $\mu \mathrm{m})$ images of the northern part and two images of the southern part of the map.

Although they have the zodiacal emission removed, ISSA fields are not calibrated so that the "zero point" corresponds to no emission, so another "background" needs to be subtracted. This background-subtraction procedure went as follows. First, the minimum value of each of the four 12.5 by 12.5 images (see Table 2) was obtained and subtracted from them. The resulting images were then used to obtain an optical extinction $\left(A_{V}\right)$ map through a process to be discussed below. At this point, after just a simple subtraction of the minimum value in each image, the north and the south $A_{V}$ maps (see Fig. 1) did not agree within the errors in the region of overlap. So, the values used for the background subtraction were iterated until the best agreement for the overlap region was found while keeping the background-subtraction constants less than $1 \mathrm{MJy} \mathrm{Sr}^{-1}$ away from the minimum flux value of the original 12.5 by 12.5 ISSA fields. Table 2 lists the values that were ultimately used for this purpose. These values resulted in a difference of $0.1 \mathrm{mag}$ between the mean in the distribution of $A_{V}$ values in the northern image and the mean in the distribution of $A_{V}$ values in the southern image. Tests using other values for background subtraction showed that the resultant extinction values did not change significantly for small (less than $\sim 1 \mathrm{MJy}^{-1}$ ) changes in the backgroundsubtraction constants. As discussed below, the offset between ISSA plates winds up being the limiting error in determining extinction from ISSA data.

The extinction map was computed from the ISSA images using a method very similar to that described by Wood et al. (1994), and references therein. Note, however, that in their study, Wood et al. (1994) used IRAS images that had not gone through a zodiacal emission removal process. They devised their own zodiacal light subtraction, which they state is not very efficient for regions near the ecliptic, like Taurus. One of the regions they study was in fact the Taurus dark cloud region itself. We believe that our extinction map is of better quality because we use ISSA images that have a more elaborate zodiacal light subtraction algorithm.

The 60 and $100 \mu \mathrm{m}$ dust temperature $T_{d}$ at each pixel in an image can be obtained by assuming that the dust in a single beam can be characterized by one temperature $\left(T_{d}\right)$, and that the observed ratio of 60 to $100 \mu \mathrm{m}$ emission is due to blackbody radiation from dust grains at $T_{d}$, modified by

TABLE 2

BACKGROUND SUBTRACTION FOR ISSA IMAGES

\begin{tabular}{ccccc}
\hline \hline & \multicolumn{2}{c}{ Minimum Flux } & \multicolumn{2}{c}{ BaCKGROUnd-SubTRACTION Constant } \\
\cline { 2 - 5 } ImAGE & $\begin{array}{c}100 \mu \mathrm{m} \\
\left(\mathrm{MJy} \mathrm{Sr}^{-1}\right)\end{array}$ & $\begin{array}{c}60 \mu \mathrm{m} \\
\left(\mathrm{MJy} \mathrm{Sr}^{-1}\right)\end{array}$ & $\begin{array}{c}100 \mu \mathrm{m} \text { Flux } \\
\left(\mathrm{MJy} \mathrm{Sr}^{-1}\right)\end{array}$ & $\begin{array}{c}60 \mu \mathrm{m} \mathrm{Flux} \\
\left(\mathrm{MJy} \mathrm{Sr}^{-1}\right)\end{array}$ \\
\hline North $^{\mathrm{a}} \ldots \ldots$ & 8.89 & -0.57 & 8.50 & -0.70 \\
South $^{\mathrm{b}} \ldots \ldots$ & 7.27 & -1.05 & 6.60 & -1.04 \\
\hline
\end{tabular}

a The north images come from the ISSA images $\mathrm{i} 311 \mathrm{~b} 3 \mathrm{~h} 0$ and $\mathrm{i} 311 \mathrm{~b} 4 \mathrm{~h} 0$, which are centered at $4^{\mathrm{h}} 36^{\mathrm{m}}, 30^{\circ}$ (B1950).

b The south images come from the ISSA images $\mathrm{i} 276 \mathrm{~b} 3 \mathrm{~h} 0$ and $\mathrm{i} 276 \mathrm{~b} 4 \mathrm{~h} 0$, which are centered at $4^{\mathrm{h}} 12^{\mathrm{m}}, 20^{\circ}(\mathrm{B} 1950)$. 
a power-law emissivity. The flux density of emission at a wavelength $\lambda_{i}$, is given by

$$
F_{i}=\left(\frac{2 h c}{\lambda_{i}^{3}} \frac{1}{e^{h c / \lambda_{i} k T_{d}}-1}\right) N_{d} \alpha \lambda_{i}^{-\beta} \Omega_{i},
$$

where $N_{d}$ is the column density of dust grains, $\beta$ is the power-law index of the dust emissivity, $\Omega_{i}$ is the solid angle at $\lambda_{i}$, and $\alpha$ is a constant of proportionality.

In order to use equation (3) to calculate the dust color temperature $\left(T_{d}\right)$ of each pixel in the image, we have to make some assumptions. The first assumption is that the dust emission is optically thin. We believe this is a safe assumption because in our maps there is not a single line of sight that could be optically thick $\left(\tau_{100}>1\right)$. In fact, the largest $\tau_{100}$ we find in our processed images is 0.002 . The second assumption we have to make is that $\Omega_{60} \cong \Omega_{100}$, which is true for all ISSA images. With these two assumptions we can then write the ratio $R$ of the flux densities at 60 and 100 $\mu \mathrm{m}$ as

$$
R=\frac{F_{60}}{F_{100}}=0.6^{-(3+\beta)}\left(\frac{e^{144 / T_{d}}-1}{e^{240 / T_{d}}-1}\right) .
$$

In order to proceed we need to assume a value of $\beta$. For now we will assume that $\beta=1$, and we will discuss the implications of this assumption later on. We constructed a look-up table with the value of $R$ calculated for a wide range of $T_{d}$, with steps in $T_{d}$ of $0.05 \mathrm{~K}$. For each pixel in the image, the table was searched for the value of $T_{d}$ that reproduces the observed 100 to $60 \mu \mathrm{m}$ flux ratio. Using the dust color temperature, we then calculate the dust optical depth for each pixel

$$
\tau_{100}=\frac{F_{\lambda}(100 \mu \mathrm{m})}{B_{\lambda}\left(\lambda, T_{d}\right)},
$$

where $B_{\lambda}\left(\lambda, T_{d}\right)$ is the Planck function and $F_{\lambda}(100 \mu \mathrm{m})$ is the observed $100 \mu$ m flux.

We use equation (5) of Wood et al. (1994) to convert from optical depth to extinction in $V$ :

$$
A_{V}=15.078\left(1-e^{-\tau_{100 / a}}\right),
$$

where $\tau_{100}$ is the optical depth and $a$ is a constant with a value of $6.413 \times 10^{-4}$. This equation relies on the work of Jarrett, Dickman, \& Herbst (1989), who present a plot (their Fig. 8) of the relation between $60 \mu \mathrm{m}$ optical depth $\left(\tau_{60}\right)$ and $A_{V}$ based on star counts. Assuming optically thin emission, Wood et al. (1994) multiply the Jarrett et al. (1989) $\tau_{60}$ values by $100 / 60$ to convert to $\tau_{100}$ and obtain equation (6), above. Thus, extinction values obtained using the ISSA images are subject to the uncertainties in the conversion equation. But, Figure 8 of Jarrett et al. (1989) shows that there is a very tight correlation between $\tau_{60}$ and $A_{V}$ for $A_{V} \leq 5 \mathrm{mag}$, implying very little uncertainty in the conversion of far-infrared optical depth to visual extinction. In the Taurus region under study in this paper, all of the extinction values measured are less than $5 \mathrm{mag}$, so we do not consider any errors caused by uncertainty in the coefficients of equation (6).

After all this processing was done, we were left with two extinction maps (a northern and a southern one) that had some overlap (see Fig. 1). The area of overlap was averaged and the north and south extinction maps were combined to produce a final image (Fig. 1) extending from R.A. of $4^{\mathrm{h}} 09^{\mathrm{m}} 00^{\mathrm{s}}$ to $4^{\mathrm{h}} 24^{\mathrm{m}} 30^{\mathrm{s}}$ (B1950) and from decl. $22^{\circ} 00^{\prime}$ to $28^{\circ} 35^{\prime}$ (B1950).

An interesting point to note is that several "elliptical holes" in the extinction appear in Figure 1. These "holes" are unphysical depressions in the extinction produced by the hot sources seen in the $60 \mu \mathrm{m}$ maps. The $60 \mu \mathrm{m}$ point sources (mostly embedded young stars) heat the dust around them and create a region where there is an excess of hot dust. This limitation, caused by the low spatial resolution of IRAS and assuming a single $T_{d}$, will then create an unphysically low extinction, when calculating the $A_{V}$ in the region near $I R A S$ point sources, using the method described above. Unfortunately, cut 2 (see Fig. 1) passes near two of these unphysically low-extinction areas. (In Figs. 4 and 6 we mark the position of the unreal dip in extinction.) These unphysical holes in $A_{V}$ are each associated with two very close IRAS point sources (one hole is produced by IRAS $0418+2654$ and IRAS $0418+2655$ and the other one by IRAS $0418+2650$ and IRAS $0419+2650$ ) inside the dark cloud B216-217 (see end of § 3.2).

As mentioned above, in order to calculate the optical depth we assumed that the dust emissivity is proportional to a power law $\left(\tau_{d} \propto \lambda^{-\beta}\right)$, with index $\beta=1$. Although studies differ in the values they find for $\beta$, there is a general agreement that the emissivity index depends on the grain's size, composition, and physical structure (Weintraub, Sandell, \& Duncan 1991). The general consensus in recent years has been that $\beta$ has a value most likely between 1 and 2 , that in the general ISM $\beta$ is close to 2 , and in denser regions with bigger grains $\beta$ is closer to 1 (Beckwith \& Sargent 1991; Mannings \& Emerson 1994; Pollack et al. 1994). Our region of interest has both lines of sight that pass through low- and high-density medium with different environments. Thus, there is no way we can use a "perfect" or "preferred" value of $\beta$, since it might be different for different lines of sight. In our case we had to choose the same value as Jarrett et al. (1989) and Wood et al. (1994), which is $\beta=1$, since we use their results to convert from $\tau_{100}$ to extinction. The errors introduced by assuming a constant $\beta$ are hard to estimate, since we do not have any way to measure how much $\beta$ changes in our region of study. We do not include these errors in the error estimate of $A_{V}$ using ISSA images (from now on $A_{V_{\text {ISSA }}}$ ), but it must be kept in mind that $\beta$ is not necessarily equal to 1 and that its value may vary for different lines of sight.

In order to estimate the pixel-to-pixel (random) errors in $A_{V_{\text {ISSA }}}$, we examined a circular area of 800 pixels centered at $4^{\mathrm{h}} 19^{\mathrm{m}}, 22^{\circ} 24^{\prime}$ (B1950), which appears to have a constant extinction. We compared pixels that are 1.5 IRAS beams apart and obtained a standard deviation in the extinction value of this region to be $0.06 \mathrm{mag}$. We use this value as an estimate of the pixel-to-pixel errors in $A_{V_{\text {ISSA }}}$.

Ultimately, though, we need an estimate of the total error in $A_{V_{\text {ISSA }}}$, not just pixel-to-pixel errors. As explained above, the north and south extinction maps give slightly different extinction values for matching pixels in the region where they overlap. By fitting a Gaussian to a histogram of the difference in $A_{V_{\text {ISSA }}}$ value between the north and the south extinction map, we find a $1 \sigma$ width of $0.11 \mathrm{mag}$. We use this value as an estimate of the $1 \sigma$ error in $A_{V_{\text {ISSA }}}$ caused by uncertainty in the zero level constants and zodiacal subtraction in the ISSA plates. Adding this "plate-to-plate" error in quadrature to the pixel-to-pixel error (previous paragraph) gives a total error in $A_{V_{\text {ISSA }}}$ of $0.12 \mathrm{mag}$. We use 
this value as an estimate of the error in $A_{V_{\text {ISSA }}}$, but we remind the reader that this error does not include any errors caused by assuming a constant $\beta$.

\section{RESULTS}

The data described above offer the opportunity to measure $A_{V}$ in four different ways: (1) using the 85 stars in cut 1 for which we have color excess data (see Table 1), (2) using 60 and $100 \mu \mathrm{m}$ ISSA images as described in $\S 2.3$, (3) using star-counting techniques on the $R$-band frames, and (4) using an optical ( $V$ and $R$ ) version of the average color excess method used by LLCB, described in more detail in $\S 3.3$.

Throughout the paper we assume that the extinction we calculate, independent of the way it was obtained, is produced by the dust associated with the Taurus dark cloud complex at a distance of $140 \pm 10 \mathrm{pc}$ (Kenyon et al. 1994). The region of Taurus we observed lies at Galactic coordinate $l \approx 172^{\circ}, b \approx-17^{\circ}$. Thus, our stars lie toward lines of sight where there is little or no dust except for that associated with the Taurus dark cloud, and we can safely assume that in the area under study, virtually all the extinction is produced by the dust associated with the Taurus dark cloud complex.

\subsection{Extinction from the Color Excess of Stars with Measured Spectral Types}

Using the color excess and position data for the stars in Table 1 that lie on cut 1 and have a distance larger than 150 pc, we construct the extinction versus declination plot shown in Figure $3 a$. This technique easily detects the rises in extinction associated with Tau M1 around decl. 23.65, L1506 around decl. 25 , B216-217 around 26.7, and the area near the IRAS cores Tau B5 and Tau B11 (hereafter Tau B5-B11) around decl. 27.5. As in the ISSA $A_{V}$ map (Fig. 1), the extinction obtained from the color excess of stars $\left(A_{V_{\mathrm{sp}}}\right)$ shows an overall rise in extinction with increasing declination. Also plotted in Figure $3 a$ are the extinction values obtained from the ISSA extinction map $\left(A_{V_{\text {ISSA }}}\right)$ for the same coordinates on the sky where there is an $A_{V_{\mathrm{sp}}}$ point. The value of each $A_{V_{\text {ISSA }}}$ point is obtained from the pixel nearest to the coordinates of each star with measured $A_{V_{\mathrm{sp}}}$ in cut 1 . The error bars, set at $\pm 0.12 \mathrm{mag}$ for each $A_{V_{\text {ISSA }}}$ point, show the total error (including pixel-to-pixel and plate-to-plate variations, but not including systematic errors) discussed above.

As a check on the assumed value of $R_{V}$, we let $R_{V}$ vary in the calculation of $A_{V_{\mathrm{sp}}}$ and then calculate $\Delta_{\text {tot }}=$ $\sum_{i=1}^{N}\left|R_{V} E_{B-V}-A_{V_{\text {ISSA }}}\right|_{i}$ (where $i$ represents the $i$ th point in the plot) for different values of $R_{V}$. We found that $R_{V} \sim 3.05$ minimizes the difference between the two curves $\left(\Delta_{\text {tot }}\right)$. This reassures us that the choice of a constant $R_{V}=3.1$ is a good assumption.

The traces of $A_{V}$ versus declination in Figure $3 a$ show a very striking similarity. Although the beam size of ISSA is $\sim 5^{\prime}$ and $A_{V_{\mathrm{sp}}}$ has a beam, due to seeing effects, of approximately $3^{\prime \prime}$ (which transform into 0.2 and 0.002 pc, respectively, at a distance of $140 \mathrm{pc}$ ), both values agree within the errors in most places. A plot of $\left(A_{V_{\mathrm{sp}}}-A_{V_{\mathrm{Iss}}}\right)$ versus declination is shown in Figure $3 b$. It can be seen that $A_{V_{\mathrm{sp}}}$ $-A_{V_{\mathrm{ISSA}}} \approx 0$, within errors, for all points not in the vicinity of a steep increase in extinction (i.e., away from dark clouds and IRAS cores). In other words, in the low-extinction regions $A_{V_{\mathrm{sp}}}$ is very similar to $A_{V_{\text {IssA }}}$, despite the great difference in the resolution of the two methods. This leads us to believe that there are no or only very small fluctuations in the extinction inside a $5^{\prime}$ beam, in low- $A_{V}$ regions. In order to put an upper limit to the magnitude of the fluctuations, we present a plot of $A_{V_{\mathrm{sp}}}-A_{V_{\text {ISSA }}}$ divided by $A_{V_{\text {ISSA }}}$, in Figure $3 c$. We discuss this plot more in $\S 4.1$.

The gently sloping solid lines in Figures $3 b$ and $3 c$ are unweighted linear fits to the points in each of the plots. Both fits have a very small, but detectable, slope $\left(0.1 \mathrm{mag} \mathrm{deg}^{-1}\right.$ for Fig. $3 b$ ). Previous studies using IRAS data have attributed the existence of gradients like these to imperfect zodiacal light subtraction (Wood et al. 1994). Moreover, the fact that the linear fit of the middle panel crosses the $A_{V_{\mathrm{sp}}}$ $-A_{V_{\text {ISSA }}}$ zero line at a declination of around $25^{\circ} .4$, near the middle of the overlap region between the northern and southern $A_{V_{\text {ISsA }}}$ (Fig. 1) map, leads us to believe that the small gradient is due to imperfect ISSA image reduction. The gradient in $A_{V_{\text {ISSA }}}$ gives a pixel-to-pixel offset of 0.008 mag beam ${ }^{-1}$, which is much less than the pixel-to-pixel random errors in $A_{V_{\text {ISSA }}}$ and much, much less than other systematic errors (e.g., constant $\beta$ ) so we do not need to correct for it.

\subsection{Star Counting}

With the help of IRAF, as described in $\S 2.1$, we located a total of 3715 stars in cut 1 and 3074 stars in cut 2, with $14.5 \leq m_{R}<18.0$, from the 1995 November $R$-band images. With this database of stellar positions we measure the extinction over the region covered by both cuts, using classical star-counting techniques. First, we subdivide each cut into a rectilinear grid of overlapping squares, and then we count the total number of stars in each square. Our ultimate goal in star counting is to obtain the extinction of the region in a way that can be compared to the other techniques used in this paper. Therefore, in order to mimic the resolution and sampling frequency of the ISSA data, we made the counting squares $5^{\prime}$ on a side (the resolution of ISSA) and the centers of the squares were separated by 1.5 (the size of an ISSA pixel).

Conventionally, measuring extinction from star counts involves comparing the integrated number of stars within a given cell toward the region of interest to a nearby reference field that is assumed to be free from extinction (Bok \& Cordwell 1973). Several assumptions need to be made in order to use this method: (1) the population of stars that are background to the region of interest does not vary substantially and is similar to the reference field, (2) the extinction $(A)$ is uniform over the count cell, and (3) the integrated surface density of stars for the reference field $\left(N_{\text {ref }}\right)$ of stars brighter than the apparent magnitude, $m$, follows an exponential law with $\log \left[N_{\text {ref }}(<m)\right]=a+b m$. The integrated surface density of stars for any other field under study, $N_{\text {on }}$, follows a similar law, $\log \left[N_{\text {on }}(<m)\right]=a+b(m-A)$.

In our case, the extinction to each square was obtained via

$$
A_{V_{\mathrm{on}}}=A_{V_{\mathrm{ref}}}+\frac{A_{V}}{A_{R}} \frac{\log \left(n_{\mathrm{ref}} / n_{\mathrm{on}}\right)}{b_{R}},
$$

where $A_{V_{\text {ref }}}$ is the extinction of the reference field, $n_{\text {ref }}$ is the number of stars in the reference field, and $n_{\text {on }}$ is the number of stars in any of the other counting cells. The quantity $b_{R}$ is 

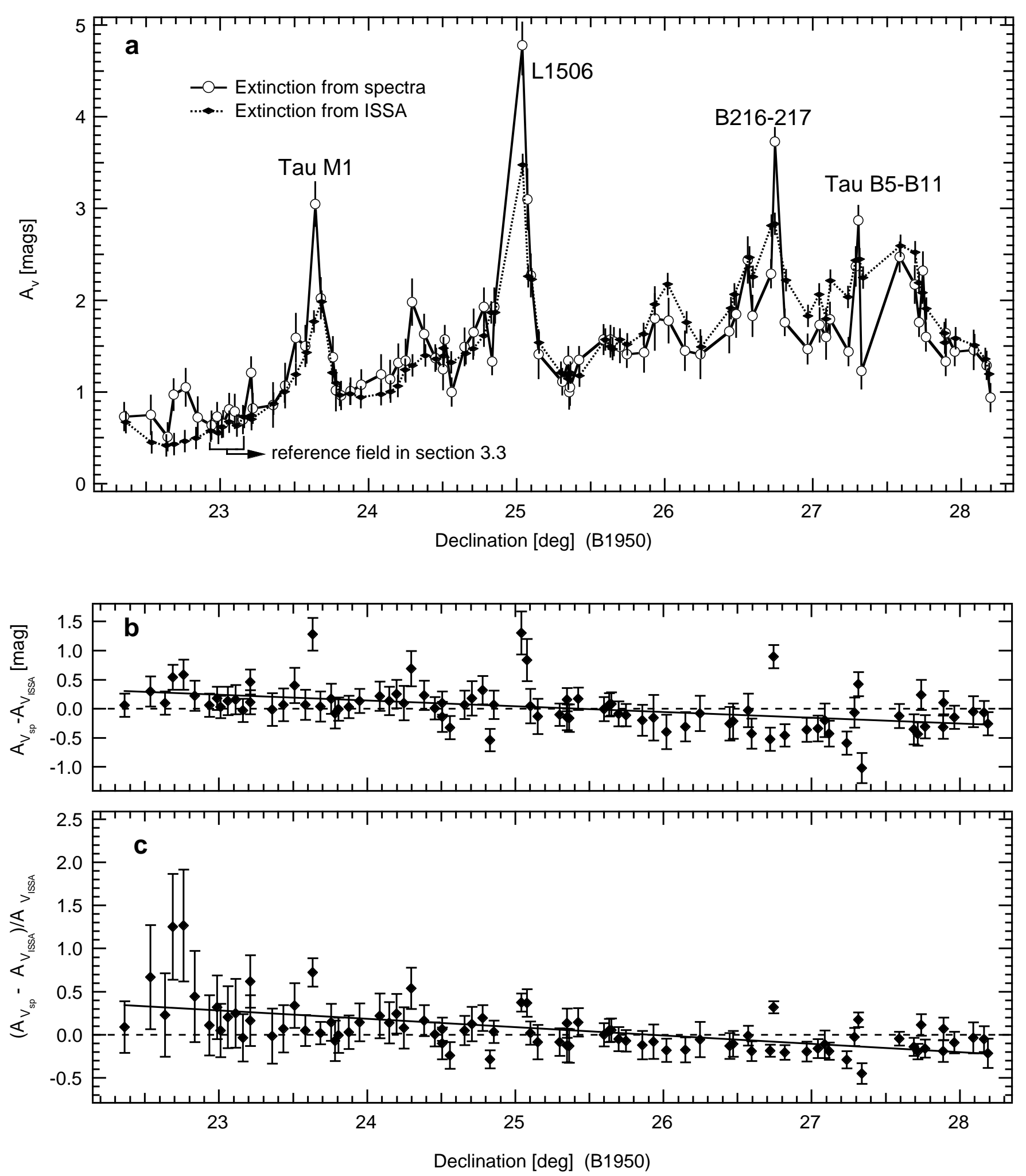

FIG. 3. - (a) Shows the extinction of every star we classified by spectral type with distances larger then 140 pc in cut 1 , as well as the extinction at the same position on the sky using the ISSA extinction map (Fig. 1). The error bars shown represent only the $1 \sigma$ random errors for each of the two methods. Also shown in $(a)$ is the region used as reference field for the extinction obtained using the technique described in $\S 3.3$. (b) Shows the difference between the two different extinction values from $(a)$. (c) Shows the difference (from $[b]$ ) divided by the ISSA extinction $\left(A_{V_{\text {ISSA }}}\right)$. The solid lines in $(b)$ and $(c)$ are the unweighted linear fits to the points in each of the two plots. These lines indicate the small gradient in extinction with declination in $A_{V_{\text {ISSA }}}$, which we believe is due to an imperfect zodiacal light subtraction of the ISSA images. 
the slope of the cumulative number of stars as a function of $R$ apparent magnitude (see assumption [3] in previous paragraph). We calculate $b_{R}$ by fitting a line to $\log \left[N_{\text {ref }}(<\right.$ $\left.\left.m_{R}\right)\right]$ for $14.5 \leq m_{R}<18.0$ and obtain a value of $0.34 \pm 0.02$. The ratio $A_{V} / A_{R}$ is the reddening law between $V$ and $R$ wavelengths, which gives the value of 1.24 ( $\mathrm{He}$ et al. 1995) used in the conversion for star counts in (Cousins) $R$ to extinction in $V$.

As mentioned above, in conventional star count studies, the reference fields are areas in the sky, close to the region under study, which have $A_{V_{\text {ref }}}=0$. This is not so in our case. Since our CCD data were not originally obtained for starcounting purposes, we did not take any images of a reference field with $A_{V_{\text {ref }}}=0$. Thus, the reference fields were chosen to be regions where we could safely assume the value of $A_{V_{\text {ref }}}$. Specifically, the reference fields were chosen by virtue of their having a spectroscopic probe star to which we derived the extinction through its color excess.

One of the major assumptions in conventional starcounting studies is that the population of background stars to the cloud is similar to that in the reference field. This is why star count studies are only done over small regions of the sky. In our case, cut 1 (the longer cut) expands $6^{\circ}$ in declination that transforms to nearly $4^{\circ}$ in Galactic latitude $(-18.8<b<-14.9)$. This span in Galactic latitude is enough to have a big effect on the surface density of stars due to Galactic variations; one detects more stars per cell, for a constant $A_{V}$, the closer one observes to the Galactic plane. (The star count Galaxy model of Reid et al. 1996 predicts that a $10 \mathrm{deg}^{2}$ field with no extinction centered at $b=-14^{\circ}, l=-172^{\circ}$ will have approximately a factor of 2 more stars than a $10 \mathrm{deg}^{2}$ field with no extinction centered at $b=-19^{\circ}, l=-172^{\circ}$.) Thus, it was imperative that we correct for Galactic changes in the stellar density. Not doing this would have resulted in an unreal drop in the derived extinction for lower Galactic latitudes. We account for the Galactic gradient by using four different, more or less evenly spaced, reference fields at different Galactic latitudes along the cut. Each of these fields has a star with measured reddening (from Table 1), which was used to estimate the extinction of the reference field in question. Thus, for example, the star count extinction located between $b=$ -18.9 and $b=-17.9$ (which transforms to $22^{\circ} 25^{\prime} \leq$ $\delta_{\mathrm{J} 2000}<23^{\circ} 56^{\prime}$ in cut 1 ) is tied to the reference field where star 011001 lies, the star count extinction in the region with $-17.9 \leq b<-16^{\circ} .9$ (which transforms to $23^{\circ} 56^{\prime} \leq$ $\delta_{\mathrm{J} 2000}<25^{\circ} 25^{\prime}$ in cut 1 ) is tied to the reference field where star 051043 lies, and so forth. The number of stars $\left(n_{\text {ref }}\right)$, the visual extinction $\left(A_{V_{\text {ref }}}\right)$, and the range in $b^{\text {II }}$, which each of the four reference fields calibrates are given in Table 3. It is important to stress that by doing this calibration we are

TABLE 3

CONTROL Fields For Star Count

\begin{tabular}{cccc}
\hline \hline \multicolumn{3}{c}{$A_{V_{\text {ref }}}$} & Range $^{\mathrm{b}}$ \\
\hline $011001 \ldots \ldots$ & 41 & $0.50 \pm 0.2$ & $-18.9 \leq b<-17.9$ \\
$051043 \ldots \ldots$ & 36 & $1.00 \pm 0.2$ & $-17.9 \leq b<-16.9$ \\
$101095 \ldots \ldots$ & 34 & $1.30 \pm 0.2$ & $-16.9 \leq b<-15^{\circ} .9$ \\
$151145 \ldots \ldots$ & 28 & $1.70 \pm 0.2$ & $-15.9 \leq b<-14.9$ \\
\hline
\end{tabular}

${ }^{a}$ Each reference field is tied to only one spectroscopic star.

${ }^{b}$ The star count extinction in the given range of $b^{\mathrm{II}}$ is calibrated using the reference field of the respective star. tying $A_{V_{\mathrm{sc}}}$ to $A_{V_{\mathrm{sp}}}$ at these four points, and thus $A_{V_{\mathrm{sc}}}$ is not totally independent of $A_{V_{\mathrm{sp}}}$. But this procedure only forces $A_{V_{\mathrm{sc}}}$ to agree in absolute value to $A_{V_{\mathrm{sp}}}$ in four points and not to give the same structure or scale through the cuts.

In our study, where observations lie primarily along a declination cut, the best way to graphically compare the extinction measured by star counting $\left(A_{V_{\text {sc }}}\right)$ and from the ISSA images $\left(A_{V_{\text {ISSA }}}\right)$ is to plot them both in an extinction versus declination plot. (Cut 1 is about $6^{\circ}$ long, but only $10^{\prime}$ wide, giving a ratio of $1: 36$ between length and width. This makes it practically impossible to show a legible figure of a star count extinction map of the cuts.) A value of the extinction was obtained for each $5^{\prime} \times 5^{\prime}$ counting box and then averaged extinction over right ascension for every point in declination, so as to produce only one value of $A_{V}$ for each declination, independent of right ascension. Constant declination slices every $5^{\prime}$ show that the variations in $A_{V_{\text {ISSA }}}$ across the $10^{\prime}$ width of each cut are not large. Most of the constant declination slices had standard deviations in $A_{V_{\text {ISSA }}}$ of less than $0.2 \mathrm{mag}$, and none exceeded $0.3 \mathrm{mag}$. Moreover, most adjacent counting boxes with the same declination differ in $A_{V_{\mathrm{sc}}}$ by less than $0.2 \mathrm{mag}$. Therefore, we are confident that we are not introducing large errors by averaging the extinction over the $\sim 10^{\prime}$ spanned by each cut in right ascension. On the other hand, by averaging over right ascension, the sensitivity to small-scale fluctuations decreases. The smoothed (averaged) extinction trace has less sensitivity in detecting fluctuations on $5^{\prime}$ scales than on $10^{\prime}$ scales, where it reaches full sensitivity. Nevertheless, averaging the extinction over right ascension does not create any disadvantage for the purpose of comparing the different ways of calculating the extinction since all the techniques are averaged over the same width.

Figure 4 shows $A_{V_{\text {sc }}}$ and $A_{V_{\text {ISSA }}}$ (now both averaged over the $\sim 10^{\prime}$ that spanned each cut in right ascension) versus declination for both cuts. ${ }^{4}$ The random errors in the star count trace are plotted for each point. The uncertainty in the number of stars in each sampling box is given by $n^{1 / 2}$ (Poisson statistics), where $n$ is the number of stars counted in the box (Bok 1937). The uncertainty in the extinction (with contributions from the uncertainty in $n, n_{\text {ref }}, A_{V_{\text {ref }}}$, and $b_{R}$ ) for each sampling box with the same declination is then averaged to give the final (plotted) error in the extinction. Like the ratio of total-to-selective extinction $\left(R_{V}\right)$, the value of $A_{V} / A_{R}$ may vary from one line of sight to the other. Also, like $R_{V}$ in $\S 2.2$, here we use the ISM average value of $A_{V} / A_{R}$. We do not take the errors caused by assuming a constant value of $A_{V} / A_{R}$ into account when we calculate the errors in $A_{V_{\mathrm{sc}}}$, as we have no way of calculating them.

It can be seen that both $A_{V_{\text {ISSA }}}$ and $A_{V_{\text {sc }}}$ show the same gross extinction structure. Both have local maxima and minima in the same places (in most cases), but not at necessarily the same value. Both traces detect rises in extinction associated with Tau M1, L1506, B216-217, and Tau B5-B11. It is clear that $A_{V_{\mathrm{sc}}}$ has more fluctuations than the $A_{V_{\text {ISSA }}}$ trace. These fluctuations are not likely to be real, as most of them are of the same magnitude as the errors in $A_{V_{\mathrm{sc}}}$. Most of the "noise" in the extinction resulted because the star count technique is very dependent on the assump-

\footnotetext{
${ }^{4}$ Notice that $A_{V_{\text {Issa }}}$ in Fig. 4 is an average over the approximately $10^{\prime}$ width of the frames taken in the optical, but still has resolution $\sim 5^{\prime}$ along the declination direction. Thus, these are not exactly the same values of $A_{V_{\text {ISSA }}}$ shown in Fig. 3, where $A_{V_{\text {ISSA }}}$ values for 1'.5 single ISSA pixels, with 5' resolution, at individual stellar positions are plotted.
} 

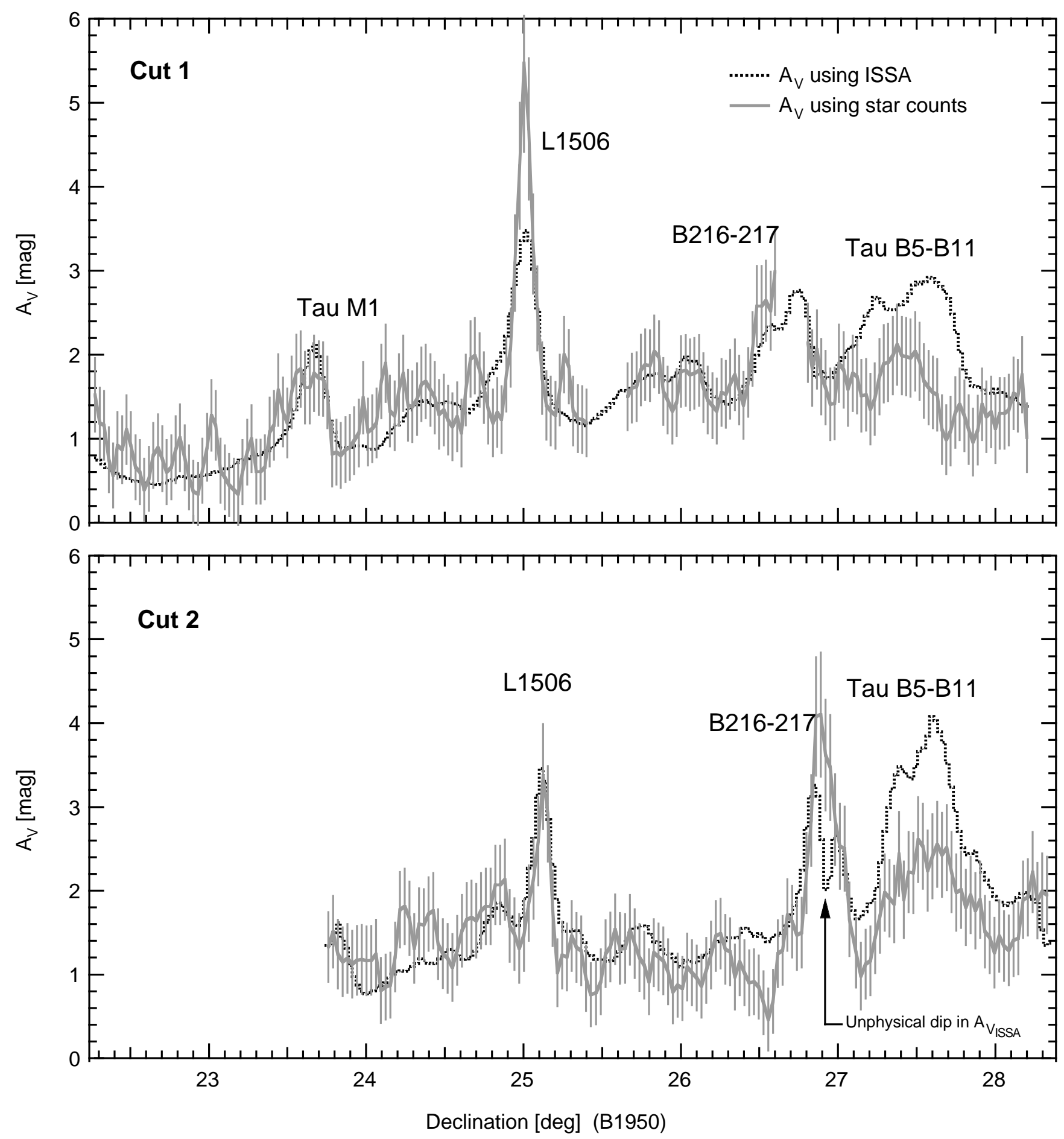

Fig. 4.-Plots of extinction vs. declination for both cut 1 and cut 2 . The solid line is the extinction obtained through star counts. The dotted line is the extinction obtained using the ISSA 60 and $100 \mu \mathrm{m}$ images. Both methods are averaged over the $10^{\prime}$ width of the cut. Breaks in the star count extinction trace are due to missing $R$-band frames. The error bars in the star count extinction represent the $1 \sigma$ error. The rise in extinction associated with the dark clouds and $I R A S$ cores is identified. The dip in the extinction peak associated with B216-217 in the $A_{V_{\text {ISsA }}}$ trace of cut 2 is due to an unreal drop in the extinction caused by the presence of $I R A S$ point source in the images.

tion of a constant background stellar surface density. Real (small) changes in the background surface density of stars (not caused by extinction) will produce unreal fluctuations in the resultant $A_{V_{\mathrm{sc}}}$.

Note the unreal dip in the cut 2 trace of $A_{V_{\text {ISSA }}}$ (see Fig. 4), caused by assuming a constant dust temperature for those lines of sight where there are $I R A S$ point sources that heat the dust around them (see $\S 2.3$ ). This shows the potentially large errors in $A_{V_{\text {ISSA }}}$ that can arise from assuming a single dust temperature $\left(T_{d}\right)$ for each line of sight. The other place where there is a significant discrepancy between $A_{V_{\text {ISSA }}}$ and $A_{V_{\mathrm{sc}}}$ is in the rise in extinction associated with Tau B5-B11 
(see Fig. 4), where the two methods disagree by more than $1 \sigma$ of the error in $A_{V_{\mathrm{sc}}}$. This discrepancy will be discussed further in $\S 4.2$.

\subsection{Extinction Measured via Average Color Excess Method}

Taking advantage of the fact that we had taken our 1995 November images in more than one broadband filter, we used the method developed by LLCB to study the extinction along both cuts in yet another way. This method consists of assuming that the color distribution of stars observed all along the cut is identical in nature to that of stars in a control field. With this assumption one can use the mean $V-R$ color of the stars in the control field to approximate the intrinsic $V-R$ color of all stars that are background to the cloud. (Note that LLCB's analysis was in the near-infrared, and they used $H-K$ colors - which have even less intrinsic variation than $V-R$ colors.) If we use the same technique as in star counting, the region under study is divided into a grid of overlapping counting boxes, and then an average of the color excess (and extinction) of the stars in each counting box is obtained.

To derive extinction from the average color excess method, we used the same sample of stars, with $14.5 \leq$ $m_{R}<18.0$, used in our star-counting study (§ 3.2). The region between declinations 22.9 and 23.22 (B1950) was used as our reference field since, as can be seen in Figure $3 a$, this region has a uniform extinction within the errors. We took an average of the extinction of the six stars for which we had spectral types and which lie inside this region, and obtained a value of $A_{V_{\text {ref }}}=0.72 \pm 0.2$. We did not divide the area under study into square sampling boxes as is usually done in star count studies (see previous section). Instead, the area under study was divided into rectangular cells, where the right ascension side of the rectangle was dictated by the frames' width $\left(\sim 10^{\prime}\right)$ and the declination side was set to be $5^{\prime}$. The centers of the rectangles were separated in declination by 1.5. This was done in order to keep the same resolution and sampling frequency in the declination direction as in the ISSA and star-counting methods (described in the previous sections). We are not sensitive to any variations in extinction within each measurement rectangle, but Figure $3 a$ suggests that such variations are very small.

The number of stars in each rectangle was counted and the color excess of each star was obtained using the formula:

$$
E_{V-R}=(V-R)-\langle V-R\rangle_{\mathrm{ref}},
$$

where $\langle V-R\rangle_{\text {ref }}$ is the mean $V-R$ color of the reference field, which in our case is $0.60 \pm 0.05$ mag. Figure 5 shows the distribution of $V-R$ colors in the reference field. ${ }^{5}$ We then apply equation (8) to each star in a counting rectangle and obtain a mean color excess to each rectangle:

$$
\left\langle E_{V-R}\right\rangle=\frac{\sum_{i}^{N}\left(E_{V-R}\right)_{i}}{N},
$$

where $N$ is the number of stars in a counting rectangle and $\left(E_{V-R}\right)_{i}$ is the color excess of the $i$ th star. The mean visual

\footnotetext{
${ }^{5}$ Notice that the width of the color distribution in Fig. 5 is significantly larger than the error in the mean $(0.05 \mathrm{mag})$. The spread in near-IR (e.g., $H-K$ ) color for a field like this would be much narrower, which is why it is preferred to use the average color excess method in the near-IR (LLCB).
}

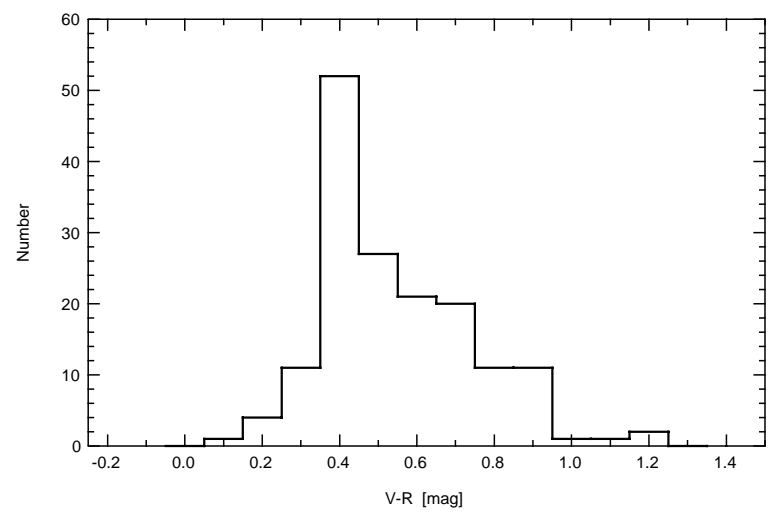

FIG. 5.-Distribution of $V-R$ colors in the reference field for the average color excess method described in $\S 3.3$.

extinction is obtained using

$$
\left\langle A_{V}\right\rangle=A_{V_{\mathrm{ref}}}+\frac{A_{V}}{E_{V-R}} \times\left\langle E_{V-R}\right\rangle,
$$

where $A_{V_{\text {ref }}}$ is the extinction of the reference field $(0.72 \mathrm{mag})$, and we use the ISM average value of 5.08 for the expression $A_{V} / E_{V-R}(\mathrm{He}$ et al. 1995). Similar to the extinction from star counts, the extinction using the average color excess (from now on $A_{V_{\mathrm{ce}}}$ ) is not totally independent of the spectral classification method. Recall that $A_{V_{\text {ref }}}$, which is used to calibrate $A_{V_{\mathrm{ce}}}$, is determined using measurements of $A_{V_{\mathrm{sp}} \text {. This }}$ calibration forces $A_{V_{\mathrm{ce}}}$ to agree in absolute value with the average of $A_{V_{\mathrm{sp}}}$ in the reference field, but it does not force $A_{V_{\mathrm{ce}}}$ to give the same structure or scale in the extinction curves throughout the cuts.

Using the average color excess technique, we are able to detect the same overall trends in extinction found from the ISSA plates, spectral analysis, and star counting (see Fig. 6). The rises in extinction due to Tau M1, L1506, B216-217, and Tau B5-B11 can be seen as well-pronounced peaks in $A_{V}$. Again, note the unreal dip in the cut 2 trace of $A_{V_{\text {ISSA }} \text {, }}$, caused by the presence of four IRAS point sources in the dark cloud B216-217 (see § 2.3). In Figure 6 we also show the random errors of each point in the $A_{V_{\mathrm{ce}}}$ trace. The measurement uncertainty in $A_{V_{\mathrm{ce}}}$ for any given counting cell is given by

$$
\sigma_{A V_{\mathrm{ce}}}=\sqrt{\sigma_{\text {ref }}^{2}+(5.08)^{2}\left(\sigma_{\text {mean }}^{2}+\sum_{1}^{N} \frac{\sigma_{(V-R)_{i}}^{2}}{N^{2}}\right)},
$$

where $\sigma_{\text {ref }}$ is the uncertainty in $A_{V_{\text {ref }}}$ (which is equal to 0.2 mag), $N$ is the number of stars in the counting cell, and $\sigma_{(V-R)_{i}}$ is the photometric error in $V-R$ of the $i$ th star inside the cell. The quantity $\sigma_{\text {mean }}$ is the error in the mean of the $V-R$ color distribution of the counting cell. The distribution of the $V-R$ colors does not have a Gaussian distribution; thus, $\sigma_{\text {mean }}$ was obtained using Monte Carlo simulations. We obtained $N_{\mathrm{mc}}$ values of $V-R$, representing the $V-R$ colors of $N_{\mathrm{mc}}$ stars in a counting cell, drawn from a distribution given by the reference field distribution (Fig. 5 ). We then computed the average $V-R$ color over these $N_{\mathrm{mc}}$ stars. The procedure was repeated 1000 times with the same $N_{\mathrm{mc}}$, from which we obtained a (Gaussian) distribution of average values. The $1 \sigma$ width of this Gaussian was 

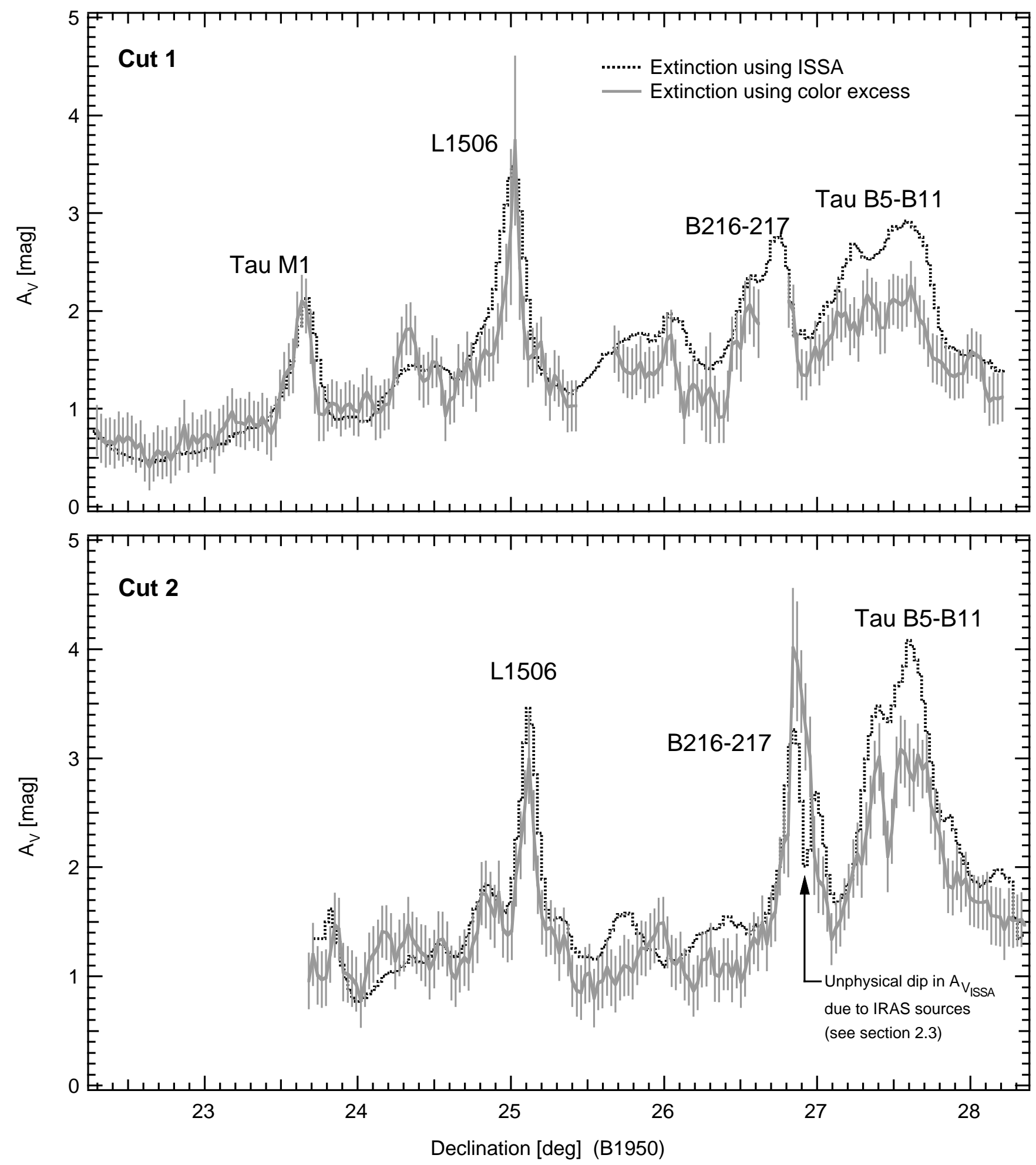

Fig. 6. - Similar to Fig. 4, but this time the solid line is the extinction obtained through the average color excess method described in $\S 3.3$. Breaks in the solid line trace in cut 1 are due to missing $R$-band frames. The error bars in the average color excess extinction represent the $1 \sigma$ error. The dip in the extinction peak associated with B216-217, in the $A_{V_{\text {ISSA }}}$ trace of cut 2 is due to an unreal drop in the extinction caused by the presence of $I R A S$ point source in the images.

then used as the value of $\sigma_{\text {mean }}$. This procedure was repeated for different values of $N_{\mathrm{mc}}$ (representing different numbers of stars inside a counting cell). We do not include the errors in $A_{V_{\mathrm{ce}}}$ caused by assuming a constant $A_{V} / E_{V-R}$ for all lines of sight.
Although $A_{V_{\mathrm{ce}}}$ and $A_{V_{\text {ISSA }}}$ agree very well for the low declination $(\delta<25.5)$ part of both cuts, their values seem to diverge for higher declinations $(\delta>25.5$, Fig. 6). This effect suggests that it may be inappropriate to assume a single average color for the whole length $\left(6^{\circ}\right)$ of our cuts. A change 
in the average $V-R$ could be due to (1) a gradient in the $\langle V-R\rangle$ caused by a greater fraction of early type stars close to the Galactic plane and/or (2) a sudden drop in the value of $\langle V-R\rangle$ in the north edge of the cuts due to the presence of a star cluster. Concerning the first point, the star count Galaxy model of Reid et al. (1996) predicts that a 10 $\mathrm{deg}^{2}$ field with no extinction centered at $b=-14^{\circ}, l=172^{\circ}$ will have approximately an average star $(V-R)$ color 0.04 mag greater than a $10 \mathrm{deg}^{2}$ field with no extinction centered at $b=-19^{\circ}, l=172^{\circ}$. A difference of $0.04 \mathrm{mag}$ in $\langle V-R\rangle$ transforms to a difference of $0.2 \mathrm{mag}$ in $A_{V_{\mathrm{ce}}}$. Thus, it is possible that at least some of the discrepancy between $A_{V_{\text {ce }}}$ and $A_{V_{\text {ISSA }}}$ for the northern parts of both cuts is due to this uncorrected effect of varying $\langle V-R\rangle$. We will discuss the possibility of a cluster in $\S 4.3$.

\section{ANALYSIS AND DISCUSSION}

\subsection{Structure in the Cloud}

Figure $3 a$ shows a striking resemblance between the extinction obtained through the use of the ISSA 60 and 100 $\mu \mathrm{m}$ images $\left(A_{V_{\text {ISSA }}}\right)$ and the extinction obtained through the color excess of individual stars that we had classified by spectral type $\left(A_{V_{\mathrm{sp}}}\right)$. Our stellar reddening (color excess) sample represents a map of the distribution of extinction $\left(A_{V_{\mathrm{sp}}}\right)$, which, although it has a "pencil beam resolution," is measured in a spatially nonuniform fashion. On the other hand, the extinction data obtained from the ISSA images is spatially continuous, with 1.5 pixels and a resolution of $5^{\prime}$ $(\sim 0.2 \mathrm{pc}$ at a distance of $140 \mathrm{pc})$. Most stars with measured $A_{V_{\mathrm{sp}}}$ are less than $5^{\prime}$ from their nearest star with measured $A_{V_{\mathrm{sp}}}$, and there are a number of cases where three and even four stars lie within $5^{\prime}$ of each other. Therefore if one were to place a $5^{\prime}$ beam anywhere along cut 1 , one would find that 1 to 4 stars would lie, in random places, inside the beam. Thus, if there were to exist big fluctuations in the dust distribution inside the $0.2 \mathrm{pc}$ beam of $I R A S$, we would see strong variations between the value of $A_{V_{\mathrm{sp}}}$ and the value of $A_{V_{\text {ISSA }}}$. Note that this fluctuation probe can only be used where there are stars that have been classified by spectra.

Using the extinction measurements shown in Figure 3, we can place an upper limit on the fluctuations in the dust distribution within a $5^{\prime}$ beam. In Figure $3 c$ we plot the difference between $A_{V_{\mathrm{sp}}}$ and $A_{V_{\text {ISSA }}}$ divided by $A_{V_{\text {IsSA }}}$ (from now on $\left.\Delta A_{V} / A_{V}\right)$ versus declination. Here the errors are obtained using the quoted errors for $A_{V_{\mathrm{sp}}}$ (see Table 1) and $A_{V_{\text {ISSA }}}(0.12 \mathrm{mag})$ and propagation of errors. One can think of $\Delta A_{V} / A_{V}$ as a measurement of the deviations in the average extinction within a fixed area of the sky. In our case the area is given by the $5^{\prime}$ beam of $A_{V_{\text {ISsA }}}$.

Figure $3 c$ has only four points with an absolute value that is more than 3 times its $1 \sigma_{i}$ error (independent of whether we correct for the small gradient in $A_{V}$ with declination), where $\sigma_{i}$ is the error of each individual point on Figure 3c. Each of these four points is associated with one of the extinction peaks created by dark clouds and IRAS cores intersecting the cut. The high values of $\Delta A_{V} / A_{V}$ could be due to two effects indistinguishable by our data; spatially unresolved steep gradients in the extinction or random fluctuations in the dust distribution inside dark clouds and IRAS cores. These possibilities will each be discussed later. All of the remaining lines of sight, in the more uniform extinction areas, have absolute values of $\Delta A_{V} / A_{V}$, which are less than 3 times their $1 \sigma_{i}$ error. If we exclude points near dramatic extinction peaks (see Fig. 3), then we do not detect any deviations from zero in $\Delta A_{V} / A_{V}$ within our sensitivity.

We can characterize our sensitivity to extinction fluctuations using the average error in $\Delta A_{V} / A_{V}$, which is given by $\sigma_{\mathrm{av}}=\sum_{i}^{N} \sigma_{i} / N$. The $1 \sigma_{\mathrm{av}}$ error in $\Delta A_{V} / A_{V}$ for points with $A_{V_{\text {ISSA }}}<0.9$ mag is 0.41 , whereas for points with $A_{V_{\text {ISSA }}} \geq 0.9$ it is 0.15 . We choose $A_{V_{\text {ISSA }}}=0.9$ as the dividing line since points with $A_{V_{\text {ISSA }}}<0.9$ mag have consistently large uncertainties. Assuming that 0.15 is the "typical" error in $\Delta A_{V} / A_{V}$ for points with $A_{V_{\text {ISSA }}} \geq 0.9$, we can then state that a real detection (using a $3 \sigma_{\mathrm{av}}$ detection limit) of sub-IRAS beam structure would be if $\Delta A_{V} / A_{V} \gtrsim 0.45$. Therefore only in places where $\Delta A_{V} / A_{V}>0.45$ can we say that there are sub-IRAS beam structures in the cloud. Any value less than 0.45 would be considered part of the "noise" and not significant enough to be considered a detection of sub $0.2 \mathrm{pc}$ structure. So, ultimately, we only detect deviations from the mean $A_{V}$ within a $0.2 \mathrm{pc}$ beam in the vicinity of $I R A S$ cores and dark clouds. Outside of those regions, deviations within a $0.2 \mathrm{pc}$ beam are limited to be less than $\Delta A_{V} / A_{V}<0.45$ (for $A_{V}>0.9$ ). For points with extinction less than $0.9 \mathrm{mag}$ the larger uncertainty in the extinction determinations means that only points with $\Delta A_{V} / A_{V} \gtrsim 1.23$ would be real fluctuation detections, and no such points are found.

\subsection{Evidence for Smooth Clouds}

In a very important study, Lada, Alves, \& Lada (1999, hereafter LAL) recently showed that smoothly varying density gradients can produce the "fluctuations" observed in extinction studies of filamentary clouds. Two studies of dust extinction in filamentary dark clouds had been conducted previous to LAL: (1) the study of IC 5146 by LLCB and (2) a study of L977 by Alves et al. (1998). Both studies find that in $1.5 \times 1.5$ cells, the dispersion of extinction measurements within a square map pixel (what LLCB name $\left.\sigma_{\text {disp }}\right)$ increases in a systematic way with the average $A_{V}$, in the range of $0<A_{V}<25 \mathrm{mag}$. Both studies conclude that the systematic trend in their $\sigma_{\text {disp }}-A_{V}$ plot, an increase of $\sigma_{\text {disp }}$ with $A_{V}$, is due to variations in the cloud structure on scales smaller than the resolution of their measurements. But neither of the studies could definitively determine the nature of the fluctuations in the extinction. This prompted LAL to study IC 5146 in the same way as LLCB, but at a higher spatial resolution $\left(30^{\prime \prime}\right)$. With the help of Monte Carlo simulations LAL conclude that the form and slope of the $\sigma_{\mathrm{disp}}-A_{V}$ relation, and hence most (if not all) of the smallscale variations in the extinction, are due to unresolved gradients in the dust distribution within the filamentary clouds. LAL note that random spatial fluctuations in the dust distribution could exist, at a very low level, in addition to the smooth gradients. They state that $\sigma_{\text {ran }} / A_{V}$ due to random fluctuations is much less than $25 \%$ at $A_{V} \sim 30 \mathrm{mag}$, which is consistent with our ( $3 \sigma)$ upper limit of $\Delta A_{V} / A_{V}=$ 0.45 at $0.9<A_{V}<3.0 \mathrm{mag}$.

Recently, Thoraval et al. (1997, hereafter TBD) observed a low- $A_{V}$ area of the IC 5146 dark cloud complex. Similar to our study, TBD concentrate their observations in a low and uniform extinction region $\left(A_{V}<5\right)$, but unlike our study, the region that TBD studied did not include a filamentary cloud. They conclude that the variations in the extinction are present at a level no larger than $\Delta A_{V} / A_{V} \sim$ 0.25 , again consistent with our $(3 \sigma)$ upper limit of $\Delta A_{V} / A_{V}=0.45$, and similar to what LAL obtain in the 
high-extinction region of IC 5146. If we exclude, in our study, the points near extinction peaks, we are left with the same results as TBD: no fluctuations on scales smaller than the resolution. These results all suggest that there is very little random spatial fluctuation in the extinction in regions of low $A_{V}$ far from extinction peaks.

Although it is not possible to definitively determine the origin of the handful of high $\Delta A_{V} / A_{V}$ we find near extinction peaks, it is more likely that they are due to unresolved steep gradients in clouds than to localized random fluctuations in the dust distribution. The filamentary dark clouds and $I R A S$ cores typically have a minor axis that is only 3 to 4 times the IRAS beam size, so some IRAS beam will undoubtedly contain a steep extinction gradient characterizing the "edge" of one of these structures. Extinction measurements using a pencil beam (e.g., $A_{V_{\mathrm{sp}}}$ ), would be able to resolve this "edge," so, near edges, large beam (e.g., $A_{V_{\text {ISSA }}}$ ) and pencil beam measurements would disagree. The results of TBD and LAL reinforce this hypothesis. Thus, we strongly believe that the high values of $\Delta A_{V} / A_{V}$ near dark clouds and IRAS cores are due to steep gradients in the extinction not resolved by the IRAS beam.

\subsection{Possible Discovery of a Previously Unknown Cluster}

While comparing our different ways of obtaining the extinction we found a peculiar discrepancy between $A_{V_{\text {ISSA }}}$ and $A_{V_{\mathrm{sc}}}$ and between $A_{V_{\mathrm{ISSA}}}$ and $A_{V_{\mathrm{ce}}}$, in the declination range from 27.2 to $28^{\circ}$ (B1950) along the two cuts (see Fig. 4). The rise in ISSA extinction in these declinations is due to the existence of a high dust concentration that Wood et al. (1994) classify as the IRAS cores Tau B5 and Tau B11 (Tau B5-B11). The trace of $A_{V_{\text {Issa }}}$ shows that the increase in the extinction associated with Tau B5-B11 is of similar or higher magnitude to the increase in extinction associated with the dark cloud B216-217, in both cuts (see Fig. 6). On the other hand, the star count extinction and the average color excess extinction show a small increase in $A_{V}$ associated with Tau B5-B11 compared to that associated with B216-217. In addition, there is no sharp decrease in the surface density of stars like the one associated with the two dark clouds in our cuts. This can be observed in both the spatial distribution of our $R$-frame stars and in the Digitized Palomar Sky Survey.

One possible explanation for the discrepancy between extinction traces is that either $A_{V_{\text {ISSA }}}$ or $A_{V_{\text {ce }}}$ and $A_{V_{\text {sc }}}$ were calculated using the wrong assumptions. It could be that the dust in the Tau B5-B11 region has different physical properties compared to the rest of the dust in the Taurus dark cloud complex, which would change the values of $\beta$ or of $A_{V} / A_{R}$ and $A_{V} / E_{V-R}$. When we calculated $A_{V_{\text {ISSA }}}, A_{V_{\mathrm{sc}}}$, and $A_{V_{\mathrm{ce}}}$, we assumed that the power-law index, $\beta$ (eq. [4]), $A_{V} / A_{R}$ (eq. [7]), and $A_{V} / E_{V-R}$ (eq. [10]) were constant for all lines of sight. We could change the value of $A_{V} / A_{R}$ (eq. [7]) from 1.24 to 1.74 in order for $A_{V_{\mathrm{sc}}}$ to be approximately equal to $A_{V_{\text {ISSA }}}$ for the lines of sight that pass through Tau B5-B11. But, the value of $A_{V} / A_{R}$ is tied to the value of $A_{V} / E_{V-R}$ by the equation $A_{V} / E_{V-R}=1 /\left(1-A_{R} / A_{V}\right)$; thus, the proposed change in $A_{V} / A_{R}$ would change $A_{V} / E_{V-R}$ from 5.08 to 2.35 , making the discrepancy between $A_{V_{\text {sc }}}$ and $A_{V_{\text {ISSA }}}$ (Fig. 6) more pronounced. An alternate possibility is that the value of $\beta$ changes from 1 to a value less than 1 for lines of sight in the region of Tau B5-B11, in that case $A_{V_{\text {ISSA }}} \approx A_{V_{\mathrm{sc}}} \approx A_{V_{\mathrm{ce}}}$. Although it is possible to have neighboring lines of sight with different dust properties, it is very unlikely (but not impossible) to have dust with $\beta<1$ in a region like Tau B5-B11, according to experimental and theoretical studies of dust properties (Weintraub et al. 1991; Pollack et al. 1994).

A more likely explanation for the discrepancy between extinction traces near Tau B5-B11 is that there is a sharp increase in the stellar distribution in the area, which we did not account for when we calculated $A_{V_{\text {sc }}}$. The existence of a previously unknown stellar cluster in the vicinity of R.A. $4^{\mathrm{h}} 19^{\mathrm{m}}$, decl. $27^{\circ} 30^{\prime}$ (B1950) could create such an increase in the number of stars in the region. Also, if the cluster is relatively young, the average stellar colors should be bluer than in the field. This can explain why the $A_{V_{\mathrm{ce}}}$ trace follows, within the error, the structure present in the $A_{V_{\text {ISSA }}}$ trace, but at a lower value, whereas the $A_{V_{\text {sc }}}$ trace decreases in extinction value without following the structure in $A_{V_{\text {IssA }}}$.

We conclude that there is a sudden increase in the stellar distribution background to Tau B5-B11 (and a change in the average $V-R$ color), which is due to a previously unknown open star cluster. ${ }^{6}$ This is more credible than a change in dust properties, since the cluster hypothesis does not require the assumption of a physically contradictory, simultaneous change in the value of $A_{V} / A_{R}$ and $A_{V} / E_{V-R}$, or an improbable value of $\beta$. We expect that further observations of the area will verify the existence of an open cluster behind Tau B5-B11.

\section{RATING THE VARIOUS METHODS}

Although we find that all four methods give generally similar results and are consistent with each other, there are some important exceptions. The discrepancies arise from different systematic errors inherent to the different techniques. For example, when calculating $A_{V_{\text {ISSA }}}$, a constant dust temperature for each line of sight was assumed. This single temperature assumption breaks down in the immediate vicinity of stars surrounded by dust. Even though it is very likely that there is dust of many different temperatures along the lines of sight to these stars, it is the hot dust that dominates the emission at 60 and $100 \mu \mathrm{m}$, resulting in an incorrect estimate of $\tau_{100}\left(\right.$ and $\left.A_{V_{\text {ISSA }}}\right)$, as we observe in the regions near IRAS sources.

The techniques that use background stellar populations to measure the extinction (i.e., star count and the average excess color method) also suffer from important systematic errors. Here the major systematic error lies in assuming a constant stellar population background to the cloud. If the region under study spans several degrees in Galactic latitude (which is our case) uncorrected gradients in the background stellar density and the average stellar color will lead to incorrect extinction measurements when using the star count method and the average excess color method, respectively. In addition, small fluctuations in the background surface stellar density can result in unreal fluctuations in the extinction.

It is important to appreciate that the techniques used in this paper are not entirely stand alone or independent methods of obtaining extinction. Even the most exact method for measuring extinction, using the color excess of individual stars with measured spectral type $\left(A_{V_{\mathrm{sp}}}\right)$, still depends on the value of $R_{V}$. Both star counting $\left(A_{V_{\mathrm{sc}}}\right)$ and

\footnotetext{
${ }^{6}$ The Lynga catalog of star clusters (Lynga 1985) does not contain a cluster in the vicinity of R.A. $4^{\mathrm{h}} 19^{\mathrm{m}}$, decl. $27^{\circ} 30^{\prime}$ (B1950).
} 


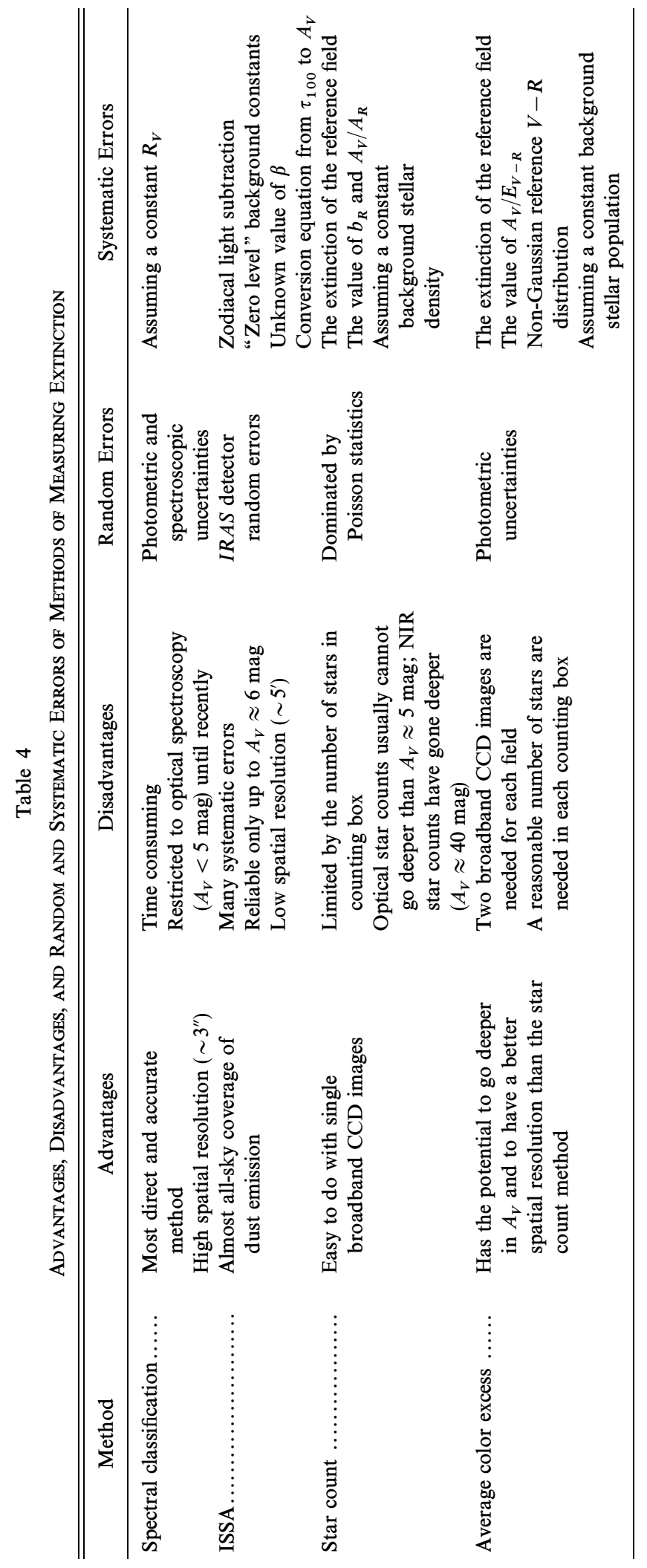


the average color excess $\left(A_{V_{\mathrm{ce}}}\right)$ technique depend on a reference field of known extinction for calibration. In this study, $A_{V_{\mathrm{sc}}}$ and $A_{V_{\mathrm{ce}}}$ are calibrated using measurements of $A_{V_{\mathrm{sp}}}$ in chosen reference fields, which means that $A_{V_{\mathrm{sc}}}$ and $A_{V_{\mathrm{ce}}}$ are then not completely independent of $A_{V_{\mathrm{sp}}}$. Note, though, that the calibration procedures only force methods to agree at a limited number of points, and they do not force them to have the same structure or scale through the cuts. $A_{V_{\text {Issa }}}$ depends on a conversion from dust opacity at $100 \mu \mathrm{m}$ to visual extinction, which ultimately relies on star count data in Jarrett et al. (1989). Thus, $A_{V_{\text {ISSA }}}$ is independent of any of the other extinction methods in this study, but it is tied to the star count data of Jarrett et al. (1989).

Table 4 outlines the advantages and disadvantages, and the random and systematic errors, of each of the four different methods of measuring extinction used in this paper.

In principle, it would seem that the best method to calculate extinction is using the color excess of individual stars with measured spectral type, but this method is not without problem. One inconvenience is the fact that $R_{V}$ could have different values for different lines of sight. But systematic errors due to unknown constants are also present in the other methods ( $\beta$ for $A_{V_{\text {ISSA }}}, A_{V} / A_{R}$ for $A_{V_{\mathrm{sc}}}$, and $A_{V} / E_{V-R}$ for $A_{V_{\mathrm{ce}}}$ ). Therefore not knowing the specific value of $R_{V}$ for every line of sight observed is not a disadvantage over the other methods. The real drawback of this technique is the large amount of time required to measure each and every star's spectrum. Thus, although using the color excess of background stars with known spectral type is the most direct and accurate way of measuring the extinction, it is a very time-consuming procedure and it measures the extinction in a spatially nonuniform fashion.

To assess the robustness of the four methods of measuring extinction used in this paper, we constructed plots of $A_{V_{\mathrm{ISSA}}}, A_{V_{\mathrm{sc}}}$, and $A_{V_{\mathrm{ce}}}$ versus $A_{V_{\mathrm{sp}}}$, at each point where all four methods can be used. We do this in order to obtain a least-square fit for each of the three remaining methods plotted against $A_{V_{\mathrm{sp}}}$. The four points with the highest extinction were not included in the fits, since these are points near the extinction peaks of dark clouds. It is clear that for these four points $A_{V_{\mathrm{sp}}}$ is larger than any of the other three methods since the $5^{\prime}$ beam of the other methods does not resolve the extinction gradients in this regions of high extinction (see $\S 4.1$ ). When we constrain the fits to have a zero intercept, we find a slope of 1 (within the errors) in all three comparisons. Thus, we believe all four methods of obtaining extinction are robust.

\section{SUMMARY AND CONCLUSIONS}

We studied the extinction of a region of Taurus in four different ways: using the color excesses of background stars for which we had spectral types, using the ISSA 60 and 100 $\mu \mathrm{m}$ images, using star counting, and using an optical ( $V$ and $R$ ) version of the average color excess technique of (Lada et al. 1994). All four give generally similar results. Therefore, any of the methods discussed above can be used to obtain reliable information about the extinction in regions where $A_{V} \lesssim 4$ mag.

We intercompared the ISSA extinction and the extinction measured using individual stars, to study the spatial fluctuations in the dust distribution. Excluding areas where there are extinction gradients due to filamentary dark clouds and IRAS cores, we do not detect any variations in the structure on scales smaller than $0.2 \mathrm{pc}$. With this result we are able to place a constraint on the magnitude of the fluctuations. We conclude that in the regions with $0.9<$ $A_{V}<3 \mathrm{mag}$, away from filamentary dark clouds and $I R A S$ cores, there are no fluctuations in the dust column density greater than $45 \%$ (at the $99.7 \%$ confidence level), on scales smaller than $0.2 \mathrm{pc}$. On the other hand, in regions of high extinction in the vicinity of dark clouds and IRAS cores, we do detect statistically significant deviations from the mean in dust column density on scales smaller than $0.2 \mathrm{pc}$. Although it is not possible to definitively determine the nature of the fluctuations with our data alone, the results of other studies (Lada et al. 1999; Thoraval et al. 1997) and ours taken together strongly favor unresolved steep gradients in clouds over random fluctuations on the dust distribution.

A discrepancy between the extinction obtained through star counting and average color excess and the rest of the techniques in the vicinity of R.A. $4^{\mathrm{h}} 19^{\mathrm{m}}$, decl. $27^{\circ} 30^{\prime}$ (B1950), leads us to believe in the existence of a previously unknown open stellar cluster in the region.

We would like to thank Lucas M. Macri very much for his great help in the photometry analysis and taking time off his observing round to take a few frames for us in 1996 October. We would also like to thank Elizabeth Barton and Warren Brown for taking the 1996 November and 1997 March frames. A special thanks goes to Perry Berlind for helping us with the acquisition of the spectral data. We would like to give special thanks to our referee Dan Clemens, as well as to Douglas Finkbeiner, for their very helpful comments and thorough critique of the paper. Also thanks to Scott J. Kenyon, George Field, Charlie Lada, and John Huchra for their helpful remarks and Ian Reid for sharing his star count Galaxy model with us.

\section{REFERENCES}

Alves, J., Lada, C., Lada, E., Kenyon, S., \& Phelps, R. 1998, ApJ, 506, 292

Arce, H. G., Goodman, A. A., Bastien, P., Manset, N., \& Sumner, M. 1998, ApJ, 499, L93

Beckwith, S. V. W., \& Sargent, A. I. 1991, ApJ, 381, 250

Bohlin, R. C., Savage, B. D., \& Drake, J. F. 1978, ApJ, 224, 132

Bok, B. J. 1937, The Distribution of Stars in Space (Chicago: Univ. Chicago Press)

Bok, B. J., \& Cordwell, C. S. 1973, in Molecules in the Galactic Environment, ed. M. A. Gordon \& L. E. Synder (New York: Wiley)

He, L., Whittet, D. C. B., Kilkenny, D., \& Spencer Jones, J. H. 1995, ApJS, 101,335

Jacoby, G. H., Hunter, D. A., \& Christian, C. A. 1984, ApJS, 56, 257

Jarret, T. H., Dickman, R. L., \& Herbst, W. 1989, ApJ, 345, 881
Kenyon, S. J., Dobrzycka, D., \& Hartman, L. 1994, AJ, 108, 1872

Kenyon, S. J., \& Hartmann, L. 1995, ApJS, 101, 117

Lada, C., Alves, J., \& Lada, E. 1999, ApJ, 512, 250 (LAL)

Lada, C. J., Lada, E. A., Clemens, D. P., \& Bally, J. 1994, ApJ, 429, 694 (LLCB)

Landolt, A. 1992, AJ, 104, 340

Lang, K. R. 1992, Astrophysical Data: Planets and Stars (New York: Springer)

Lynga, G. 1985, in Proc. IAU Symp. 106, Milky Way Galaxy, ed. H. van Woerden, R. J. Allen, \& W. B. Burton (Dordrecht: Kluwer), 143

Mannings, V., \& Emerson, J. P. 1994, MNRAS, 267, 361

Mihalas, D., \& Binney, J. 1981, Galactic Astronomy (San Francisco: Freeman) 
O'Conell, R. W. 1973, AJ, 78, 1074

Pollack, J. B., Hollenbach, D., Simonelli, D. P., Roush, T., \& Fong, W.

Savage, B. D., \& Mathis, J. S. 1979, ARA\&A, 17, 73

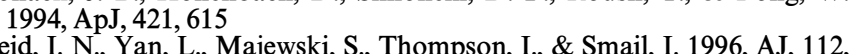

Thoraval, S., Boissé, P., \& Duvert, G. 1997, A\&A, 319, 948 (TBD)

Vrba, F. J., \& Rydgren, A. E. 1985, AJ, 90, 1490

1472 , Yan, L., Majewski, S., Thompson, I., \& Smail, I. 1996, AJ, 112

Weintraub, D. A., Sandell, G., \& Duncan, W. D. 1991, ApJ, 382, 270

Wood, D., Myers, P. C., \& Daugherty, D. A. 1994, ApJS, 95, 457

Note added in proof.-The results of this study were compared to the all-sky reddening map of D. J. Schlegel, D. P. Finkbeiner, \& M. Davis (ApJ, 500, 525, [1998]) and are published in H. G. Arce \& A. A. Goodman (ApJ, 512, L135 [1999]). 